Supporting Information for:

\title{
Monocyclopentadienylhydride derivatives of ruthenium: stereoselective proton transfer and proton-hydride exchange in an extremely short dihydrogen bond
}

Ester Cayuela, Félix A. Jalón*, Blanca R. Manzano, Gustavo Espino, Walter Weissensteiner, Kurt Mereiter

Table S1. Crystal data and structure refinement for $\mathrm{CpRuH}\left(\mathrm{PP}^{\mathrm{Ph}} \mathrm{PF}\right),(4)$.

Table S2. Atomic coordinates and equivalent isotropic displacement parameters for $\mathrm{CpRuH}\left(\mathrm{PP}^{\mathrm{Ph}} \mathrm{PF}\right),(\mathbf{4})$.

Table S3. Hydrogen coordinates and isotropic displacement parameters for $\mathrm{CpRuH}\left(\mathrm{PP}{ }^{\mathrm{Ph}} \mathrm{PF}\right)$, (4).

Table S4. Anisotropic displacement parameters for $\mathrm{CpRuH}\left(\mathrm{PP}^{\mathrm{Ph}} \mathrm{PF}\right)$, (4).

Table S5. Bond length and angles for $\mathrm{CpRuH}\left(\mathrm{PP}^{\mathrm{Ph}} \mathrm{PF}\right)$, (4).

Figure S1. ORTEP representation of the molecular structure of $\mathrm{CpRuH}\left(\mathrm{PP}^{\mathrm{Ph}} \mathrm{PF}\right),(\mathbf{4})$.

Table S6. Crystal data and structure refinement for $\mathrm{CpRu}\left(\mathrm{CF}_{3} \mathrm{CO}_{2}\right)\left(\mathrm{PP}^{\mathrm{Ph}} \mathrm{PF}\right),(\mathbf{1 3})$.

Table S7. Atomic coordinates and equivalent isotropic displacement parameters for $\mathrm{CpRu}\left(\mathrm{CF}_{3} \mathrm{CO}_{2}\right)\left(\mathrm{PP}^{\mathrm{Ph}} \mathrm{PF}\right),(\mathbf{1 3})$.

Table S8. Hydrogen coordinates and isotropic displacement parameters for $\mathrm{CpRu}\left(\mathrm{CF}_{3} \mathrm{CO}_{2}\right)\left(\mathrm{PP}^{\mathrm{Ph}} \mathrm{PF}\right),(\mathbf{1 3})$.

Table S9. Anisotropic displacement parameters for $\mathrm{CpRu}\left(\mathrm{CF}_{3} \mathrm{CO}_{2}\right)\left(\mathrm{PP}^{\mathrm{Ph}} \mathrm{PF}\right),(\mathbf{1 3})$.

Table S10. Bond length and angles for $\mathrm{CpRu}\left(\mathrm{CF}_{3} \mathrm{CO}_{2}\right)\left(\mathrm{PP}^{\mathrm{Ph}} \mathrm{PF}\right),(13)$.

Figure. S2. Thermal ellipsoid plot (20\% ellipsoids) of $\mathrm{CpRu}\left(\mathrm{CF}_{3} \mathrm{CO}_{2}\right)\left(\mathrm{PP}^{\mathrm{Ph}} \mathrm{PF}\right),(\mathbf{1 3})$.

${ }^{13} \mathrm{C}$ NMR information for ligands and complexes 1-6 
Table S1. Crystal data and structure refinement for $\mathrm{CpRuH}\left(\mathrm{PP}^{\mathrm{Ph}} \mathrm{PF}\right),(4)$.

Identification code

Empirical formula

Formula weight

Temperature

Wavelength

Crystal system, space group

Unit cell dimensions

Volume

Z, Calculated density

Absorption coefficient

$\mathrm{F}(000)$

Crystal size

Diffractometer

Scan type / width / speed

Theta range for data collection

Index ranges

Reflections collected / unique

Completeness to theta $=30.00$

Absorption correction

Transmission factors

Structure solution

Refinement method

Data / restraints / parameters

Goodness-of-fit on $\mathrm{F}^{2}$

Final R indices [I $>2 \operatorname{sigma}(\mathrm{I})]$

$\mathrm{R}$ indices (all data)

Largest diff. peak and hole 511weimx 3

C42 H38 Fe P2 Ru

761.58

297(2) K

$0.71073 \AA$

triclinic, $\mathrm{P}-1$ (no. 2)

$a=11.318(4) \AA \quad \alpha=93.94(2) \mathrm{deg}$.

$\mathrm{b}=11.675(4) \AA \quad \beta=97.22(2) \mathrm{deg}$.

$c=13.253(5) \AA \quad \gamma=106.93(2) \mathrm{deg}$.

1651.8(9) $\AA^{3}$

2, $1.531 \mathrm{Mg} / \mathrm{m}^{3}$

$1.024 \mathrm{~mm}^{-1}$

780

$0.20 \times 0.22 \times 0.36 \mathrm{~mm}$, orange prism

Bruker SMART CCD platform type 3-circle (sealed X-ray tube, Mo K $\alpha$ radiation, graphite monochr., det.dist. $44.50 \mathrm{~mm}, 512 \times 512$ pixels) $\omega$-scan frames $/ \Delta \omega=0.3^{\circ} / 25 \mathrm{sec}$. per frame full sphere data collection, 4 x 606 frames 2.21 to $30.00 \mathrm{deg}$.

$-15<=\mathrm{h}<=15,-16<=\mathrm{k}<=16,-18<=\mathrm{l}<=18$

$24309 / 9439$ [R(int $)=0.027, \mathrm{R}($ sigma $)=0.015]$

$98.1 \%$

Multi-scan (program SADABS; Sheldrick, 1996)

$0.86-0.71$

Direct methods (program SHELXS97)

Full-matrix least-squares on $\mathrm{F}^{2}$ (prg SHELXL97)

9439 / 0 / 419

1.044

$\mathrm{R} 1=0.0329, \mathrm{wR} 2=0.0793 \quad(7923$ data $)$

$\mathrm{R} 1=0.0431, w \mathrm{R} 2=0.0880 \quad$ (9439 data)

0.66 and -0.97 e $\AA^{-3}$

$\mathrm{R} 1=\Sigma|| \mathrm{F}_{\mathrm{o}}|-| \mathrm{F}_{\mathrm{c}}|/ \Sigma| \mathrm{F}_{\mathrm{o}} \mid, \mathrm{wR} 2=\left[\Sigma\left(\mathrm{w}\left(\mathrm{F}_{\mathrm{o}}^{2}-\mathrm{F}_{\mathrm{c}}^{2}\right)^{2}\right) / \Sigma\left(\mathrm{w}\left(\mathrm{F}_{\mathrm{o}}^{2}\right)^{2}\right)\right]^{1 / 2}$ 
Table S2. Atomic coordinates ( x $\left.10^{4}\right)$ and equivalent isotropic displacement parameters $\left(\AA^{2}\right.$ $\mathrm{x} 10^{3}$ ) for $\mathrm{CpRuH}\left(\mathrm{PP}^{\mathrm{Ph}} \mathrm{PF}\right),(4) . \mathrm{U}_{\mathrm{eq}}$ is defined as one third of the trace of the orthogonalized $\mathrm{U}_{\mathrm{ij}}$ tensor.

\begin{tabular}{|c|c|c|c|c|}
\hline & $\mathrm{x}$ & $\mathrm{y}$ & $\mathrm{z}$ & $\mathrm{U}_{\text {eq }}$ \\
\hline $\mathrm{Ru}$ & $744.4(2)$ & $2477.9(1)$ & $1947.3(1)$ & $34(1)$ \\
\hline $\mathrm{Fe}$ & $4888.6(3)$ & $2114.2(3)$ & $2752.5(3)$ & $37(1)$ \\
\hline $\mathrm{P}(1)$ & $2374.5(5)$ & $3193.7(4)$ & $3189.7(4)$ & $32(1)$ \\
\hline$P(2)$ & $2008.9(5)$ & $3271.2(4)$ & $841.9(4)$ & $31(1)$ \\
\hline$C(1)$ & $3441(2)$ & $2792(2)$ & $843(2)$ & $33(1)$ \\
\hline$C(2)$ & $3052(2)$ & $1431(2)$ & $459(2)$ & $41(1)$ \\
\hline$C(3)$ & $4049(2)$ & $793(2)$ & $630(2)$ & $46(1)$ \\
\hline$C(11)$ & $4425(2)$ & $660(2)$ & $1725(2)$ & $44(1)$ \\
\hline$C(12)$ & $3661(2)$ & $439(2)$ & $2508(2)$ & $50(1)$ \\
\hline$C(13)$ & $4444(3)$ & $573(2)$ & $3468(2)$ & $60(1)$ \\
\hline$C(14)$ & $5683(3)$ & $846(3)$ & $3268(2)$ & $63(1)$ \\
\hline$C(15)$ & $5683(2)$ & $896(2)$ & $2207(2)$ & $54(1)$ \\
\hline$C(21)$ & $4260(2)$ & $3204(2)$ & $1873(2)$ & $32(1)$ \\
\hline$C(22)$ & $3922(2)$ & $3330(2)$ & $2885(2)$ & $33(1)$ \\
\hline$C(23)$ & $5058(2)$ & $3704(2)$ & $3600(2)$ & $41(1)$ \\
\hline$C(24)$ & $6075(2)$ & $3835(2)$ & $3053(2)$ & $46(1)$ \\
\hline C (25) & $5598(2)$ & $3547(2)$ & $1999(2)$ & $42(1)$ \\
\hline$C(31)$ & $2371(2)$ & $2407(2)$ & $4347(2)$ & $41(1)$ \\
\hline$C(32)$ & $3052(3)$ & $2985(3)$ & $5285(2)$ & $56(1)$ \\
\hline$C(33)$ & 3097 (3) & $2354(4)$ & $6125(2)$ & $71(1)$ \\
\hline$C(34)$ & $2457(3)$ & $1142(4)$ & $6040(3)$ & $75(1)$ \\
\hline$C(35)$ & $1749(3)$ & $570(3)$ & $5132(3)$ & $69(1)$ \\
\hline$C(36)$ & $1702(3)$ & $1204(2)$ & $4283(2)$ & $51(1)$ \\
\hline$C(41)$ & $2556(2)$ & $4734(2)$ & $3746(2)$ & $40(1)$ \\
\hline$C(42)$ & $3477(2)$ & $5733(2)$ & $3558(2)$ & $44(1)$ \\
\hline$C(43)$ & $3466(3)$ & $6890(2)$ & $3873(2)$ & $59(1)$ \\
\hline$C(44)$ & $2537(4)$ & $7052(3)$ & $4381(3)$ & $74(1)$ \\
\hline C (45) & $1632(4)$ & $6068(4)$ & $4595(3)$ & $84(1)$ \\
\hline$C(46)$ & $1646(3)$ & $4910(3)$ & $4292(3)$ & $65(1)$ \\
\hline C (51) & $2653(2)$ & $4921(2)$ & $979(2)$ & $37(1)$ \\
\hline$C(52)$ & $3892(2)$ & $5544(2)$ & $954(2)$ & $46(1)$ \\
\hline$C(53)$ & $4319(3)$ & $6795(2)$ & $1129(2)$ & $59(1)$ \\
\hline$C(54)$ & $3494(4)$ & $7429(2)$ & $1299(2)$ & $66(1)$ \\
\hline C (55) & $2260(4)$ & $6823(2)$ & $1312(2)$ & $65(1)$ \\
\hline$C(56)$ & $1839(3)$ & $5577(2)$ & $1164(2)$ & $49(1)$ \\
\hline$C(61)$ & $1358(2)$ & $2950(2)$ & $-525(2)$ & $37(1)$ \\
\hline$C(62)$ & $1687(3)$ & $3774(2)$ & $-1226(2)$ & $57(1)$ \\
\hline$C(63)$ & $1204(4)$ & $3477(3)$ & $-2251(2)$ & $74(1)$ \\
\hline$C(64)$ & $397(3)$ & $2355(3)$ & $-2602(2)$ & $66(1)$ \\
\hline$C(65)$ & $63(3)$ & $1523(3)$ & $-1921(2)$ & $56(1)$ \\
\hline$C(66)$ & $528(2)$ & $1820(2)$ & $-891(2)$ & $46(1)$ \\
\hline$C(71)$ & $-1108(3)$ & $1225(3)$ & $2109(3)$ & $68(1)$ \\
\hline$C(72)$ & $-1222(2)$ & $1655(4)$ & $1152(3)$ & $72(1)$ \\
\hline$C(73)$ & $-1014(3)$ & $2902(3)$ & $1326(3)$ & $69(1)$ \\
\hline$C(74)$ & $-786(3)$ & $3234(3)$ & $2374(3)$ & $62(1)$ \\
\hline$C(75)$ & $-831(2)$ & $2194(3)$ & $2861(2)$ & $61(1)$ \\
\hline
\end{tabular}




\section{S4}

Table S3. Hydrogen coordinates ( x 10 $)$ and isotropic displacement parameters $\left(\AA^{2} \times 10^{3}\right.$ ) for $\mathrm{CpRuH}\left(\mathrm{PP}^{\mathrm{Ph}} \mathrm{PF}\right),(\mathbf{4})$.

\begin{tabular}{|c|c|c|c|c|}
\hline & $\mathrm{x}$ & $\mathrm{Y}$ & $\mathrm{z}$ & $U_{\text {eq }}$ \\
\hline $\mathrm{H}(1 \mathrm{RU})$ & $1280(20)$ & $1430(20)$ & 1905 (19) & 40 \\
\hline $\mathrm{H}(1)$ & 3907 & 3224 & 337 & 40 \\
\hline $\mathrm{H}(2 \mathrm{~A})$ & 2355 & 1020 & 790 & 49 \\
\hline $\mathrm{H}(2 \mathrm{~B})$ & 2750 & 1334 & -269 & 49 \\
\hline $\mathrm{H}(3 \mathrm{~A})$ & 3737 & -1 & 247 & 55 \\
\hline $\mathrm{H}(3 \mathrm{~B})$ & 4783 & 1241 & 359 & 55 \\
\hline $\mathrm{H}(12)$ & 2794 & 240 & 2410 & 60 \\
\hline $\mathrm{H}(13)$ & 4185 & 495 & 4105 & 72 \\
\hline $\mathrm{H}(14)$ & 6388 & 973 & 3756 & 75 \\
\hline $\mathrm{H}(15)$ & 6384 & 1056 & 1878 & 65 \\
\hline $\mathrm{H}(23)$ & 5116 & 3837 & 4307 & 49 \\
\hline $\mathrm{H}(24)$ & 6915 & 4071 & 3338 & 56 \\
\hline $\mathrm{H}(25)$ & 6074 & 3576 & 1473 & 51 \\
\hline $\mathrm{H}(32)$ & 3481 & 3804 & 5346 & 67 \\
\hline $\mathrm{H}(33)$ & 3560 & 2747 & 6747 & 85 \\
\hline $\mathrm{H}(34)$ & 2505 & 713 & 6602 & 90 \\
\hline $\mathrm{H}(35)$ & 1300 & -242 & 5081 & 83 \\
\hline $\mathrm{H}(36)$ & 1215 & 813 & 3668 & 61 \\
\hline $\mathrm{H}(42)$ & 4114 & 5631 & 3215 & 53 \\
\hline $\mathrm{H}(43)$ & 4090 & 7555 & 3738 & 70 \\
\hline $\mathrm{H}(44)$ & 2519 & 7825 & 4581 & 89 \\
\hline $\mathrm{H}(45)$ & 1005 & 6177 & 4946 & 100 \\
\hline $\mathrm{H}(46)$ & 1040 & 4250 & 4456 & 78 \\
\hline $\mathrm{H}(52)$ & 4446 & 5122 & 817 & 55 \\
\hline $\mathrm{H}(53)$ & 5159 & 7203 & 1133 & 71 \\
\hline $\mathrm{H}(54)$ & 3773 & 8266 & 1404 & 80 \\
\hline $\mathrm{H}(55)$ & 1704 & 7252 & 1421 & 78 \\
\hline $\mathrm{H}(56)$ & 1004 & 5175 & 1189 & 59 \\
\hline $\mathrm{H}(62)$ & 2240 & 4536 & -1001 & 68 \\
\hline $\mathrm{H}(63)$ & 1427 & 4043 & -2709 & 88 \\
\hline $\mathrm{H}(64)$ & 78 & 2157 & -3294 & 79 \\
\hline $\mathrm{H}(65)$ & -479 & 758 & -2155 & 67 \\
\hline $\mathrm{H}(66)$ & 284 & 1255 & -436 & 56 \\
\hline $\mathrm{H}(71)$ & -1201 & 428 & 2222 & 81 \\
\hline $\mathrm{H}(72)$ & -1403 & 1198 & 517 & 86 \\
\hline $\mathrm{H}(73)$ & -1027 & 3419 & 824 & 82 \\
\hline $\mathrm{H}(74)$ & -631 & 4008 & 2697 & 74 \\
\hline $\mathrm{H}(75)$ & -699 & 2160 & 3563 & 73 \\
\hline
\end{tabular}

$\mathrm{H}(1 \mathrm{Ru})$ is the metal bound hydride hydrogen which was refined in $\mathrm{x}, \mathrm{y}, \mathrm{z}$. All other hydrogen atoms were inserted in idealized positions and were refined riding with the atoms to which they were bonded. All $\mathrm{H}$ atoms had $\mathrm{U}_{\mathrm{iso}}=\mathrm{U}_{\text {eq }} \times 1.2$ of their carrier atoms. 
Table S4. Anisotropic displacement parameters $\left(\AA^{2} \times 10^{3}\right)$ for $\mathrm{CpRuH}\left(\mathrm{PP}^{\mathrm{Ph}} \mathrm{PF}\right),(4)$. The anisotropic displacement factor exponent takes the form: $-2 \pi^{2}\left[h^{2} a^{* 2} U_{11}+\ldots+2 h\right.$ $\left.\mathrm{k} \mathrm{a}{ }^{*} \mathrm{~b}^{*} \mathrm{U}_{12}\right]$.

\begin{tabular}{|c|c|c|c|c|c|c|}
\hline & $\mathrm{U}_{11}$ & $\mathrm{U}_{22}$ & $\mathrm{U}_{33}$ & $\mathrm{U}_{23}$ & $\mathrm{U}_{13}$ & $\mathrm{U}_{12}$ \\
\hline $\mathrm{Ru}$ & $33(1)$ & $35(1)$ & $33(1)$ & $5(1)$ & $6(1)$ & $10(1)$ \\
\hline $\mathrm{Fe}$ & $34(1)$ & $32(1)$ & $45(1)$ & $1(1)$ & $2(1)$ & $12(1)$ \\
\hline $\mathrm{P}(1)$ & $36(1)$ & $32(1)$ & $29(1)$ & $4(1)$ & $6(1)$ & $10(1)$ \\
\hline$P(2)$ & $39(1)$ & $27(1)$ & $29(1)$ & $3(1)$ & $6(1)$ & $11(1)$ \\
\hline$C(1)$ & $40(1)$ & $28(1)$ & $33(1)$ & $1(1)$ & $10(1)$ & $11(1)$ \\
\hline$C(2)$ & $46(1)$ & $34(1)$ & $42(1)$ & $-7(1)$ & $2(1)$ & $15(1)$ \\
\hline$C(3)$ & 47 (1) & $36(1)$ & $56(1)$ & $-8(1)$ & $7(1)$ & $17(1)$ \\
\hline$C(11)$ & $43(1)$ & $29(1)$ & $61(2)$ & $-2(1)$ & $2(1)$ & $15(1)$ \\
\hline$C(12)$ & 49 (1) & $30(1)$ & $70(2)$ & $7(1)$ & $9(1)$ & $11(1)$ \\
\hline$C(13)$ & $82(2)$ & $46(1)$ & $60(2)$ & $16(1)$ & $11(1)$ & $29(1)$ \\
\hline$C(14)$ & $67(2)$ & $58(2)$ & $70(2)$ & $0(1)$ & $-11(1)$ & $38(1)$ \\
\hline$C(15)$ & $48(1)$ & $48(1)$ & $72(2)$ & $-6(1)$ & $2(1)$ & $27(1)$ \\
\hline$C(21)$ & $33(1)$ & $25(1)$ & $37(1)$ & $0(1)$ & $6(1)$ & $7(1)$ \\
\hline$C(22)$ & $34(1)$ & $28(1)$ & $36(1)$ & $0(1)$ & $4(1)$ & $8(1)$ \\
\hline$C(23)$ & $41(1)$ & $36(1)$ & $40(1)$ & $-5(1)$ & $-1(1)$ & $8(1)$ \\
\hline$C(24)$ & $36(1)$ & $40(1)$ & $56(2)$ & $-3(1)$ & $1(1)$ & $5(1)$ \\
\hline$C(25)$ & $36(1)$ & $35(1)$ & $52(1)$ & $1(1)$ & $12(1)$ & $5(1)$ \\
\hline$C(31)$ & $42(1)$ & $49(1)$ & $36(1)$ & $12(1)$ & $9(1)$ & $16(1)$ \\
\hline$C(32)$ & $57(2)$ & $69(2)$ & $39(1)$ & $13(1)$ & $7(1)$ & $13(1)$ \\
\hline$C(33)$ & $70(2)$ & $105(3)$ & $39(2)$ & $26(2)$ & $9(1)$ & $27(2)$ \\
\hline$C(34)$ & $80(2)$ & $106(3)$ & $62(2)$ & $51(2)$ & $27(2)$ & $47(2)$ \\
\hline$C(35)$ & $76(2)$ & $66(2)$ & $79(2)$ & $40(2)$ & $30(2)$ & $28(2)$ \\
\hline$C(36)$ & $57(1)$ & 48 (1) & $52(2)$ & $17(1)$ & $13(1)$ & $17(1)$ \\
\hline$C(41)$ & $47(1)$ & $40(1)$ & $33(1)$ & $-1(1)$ & $4(1)$ & $18(1)$ \\
\hline$C(42)$ & $58(1)$ & $38(1)$ & $36(1)$ & $0(1)$ & $3(1)$ & $17(1)$ \\
\hline$C(43)$ & $83(2)$ & $38(1)$ & $50(2)$ & $-4(1)$ & $-7(1)$ & $20(1)$ \\
\hline$C(44)$ & $93(2)$ & $57(2)$ & $74(2)$ & $-24(2)$ & $-12(2)$ & $41(2)$ \\
\hline$C(45)$ & $78(2)$ & $88(2)$ & $91(3)$ & $-32(2)$ & $15(2)$ & $43(2)$ \\
\hline$C(46)$ & $60(2)$ & $63(2)$ & $72(2)$ & $-14(1)$ & $22(1)$ & $19(1)$ \\
\hline$C(51)$ & $56(1)$ & $28(1)$ & $26(1)$ & $3(1)$ & $7(1)$ & $13(1)$ \\
\hline$C(52)$ & $63(1)$ & $31(1)$ & $42(1)$ & $3(1)$ & $18(1)$ & $9(1)$ \\
\hline$C(53)$ & $89(2)$ & $32(1)$ & $48(2)$ & $4(1)$ & $24(1)$ & $2(1)$ \\
\hline$C(54)$ & $121(3)$ & $28(1)$ & $50(2)$ & $5(1)$ & $19(2)$ & $20(1)$ \\
\hline$C(55)$ & $108(3)$ & $45(1)$ & $55(2)$ & $6(1)$ & $13(2)$ & $44(2)$ \\
\hline$C(56)$ & $67(2)$ & $41(1)$ & $46(1)$ & $4(1)$ & $5(1)$ & $26(1)$ \\
\hline$C(61)$ & $46(1)$ & $37(1)$ & $31(1)$ & $1(1)$ & $4(1)$ & $16(1)$ \\
\hline$C(62)$ & $85(2)$ & $43(1)$ & $37(1)$ & $4(1)$ & $8(1)$ & $12(1)$ \\
\hline$C(63)$ & $120(3)$ & $65(2)$ & $35(1)$ & $8(1)$ & $7(2)$ & $28(2)$ \\
\hline$C(64)$ & $83(2)$ & $76(2)$ & $39(1)$ & $-8(1)$ & $-6(1)$ & $34(2)$ \\
\hline$C(65)$ & $54(1)$ & $58(2)$ & $50(2)$ & $-14(1)$ & $-6(1)$ & $18(1)$ \\
\hline$C(66)$ & $49(1)$ & $44(1)$ & $45(1)$ & $2(1)$ & $3(1)$ & $14(1)$ \\
\hline$C(71)$ & $41(1)$ & $58(2)$ & $107(3)$ & $19(2)$ & $26(2)$ & $9(1)$ \\
\hline$C(72)$ & $35(1)$ & $100(3)$ & $64(2)$ & $-13(2)$ & $3(1)$ & $4(1)$ \\
\hline$C(73)$ & $45(1)$ & $101(2)$ & $67(2)$ & $32(2)$ & $5(1)$ & $29(2)$ \\
\hline$C(74)$ & 49 (1) & $68(2)$ & $78(2)$ & $3(2)$ & $16(1)$ & $31(1)$ \\
\hline$C(75)$ & $44(1)$ & $92(2)$ & $56(2)$ & $22(2)$ & $21(1)$ & $26(1)$ \\
\hline
\end{tabular}


Table S5. Bond lengths $[\AA ̊]$ and angles [deg] for $\mathrm{CpRuH}\left(\mathrm{PP}^{\mathrm{Ph}} \mathrm{PF}\right),(\mathbf{4})$.

\begin{tabular}{|c|c|c|c|}
\hline \multicolumn{2}{|c|}{ Bond distances } & $\mathrm{C}(24)-\mathrm{H}(24)$ & 0.93 \\
\hline & & $\mathrm{C}(25)-\mathrm{H}(25)$ & 0.93 \\
\hline Ru-C (71) & $2.224(3)$ & $C(31)-C(36)$ & $1.379(3)$ \\
\hline $\mathrm{Ru}-\mathrm{P}(1)$ & $2.2277(12)$ & $C(31)-C(32)$ & $1.391(4)$ \\
\hline $\mathrm{Ru}-\mathrm{P}(2)$ & $2.2348(11)$ & $C(32)-C(33)$ & $1.380(4)$ \\
\hline Ru-C ( 75) & $2.238(3)$ & $\mathrm{C}(32)-\mathrm{H}(32)$ & 0.93 \\
\hline Ru-C ( 72) & $2.239(3)$ & $C(33)-C(34)$ & $1.379(5)$ \\
\hline $\mathrm{Ru}-\mathrm{C}(73)$ & $2.259(3)$ & $\mathrm{C}(33)-\mathrm{H}(33)$ & 0.93 \\
\hline $\mathrm{Ru}-\mathrm{C}(74)$ & $2.273(3)$ & $C(34)-C(35)$ & $1.368(5)$ \\
\hline \multirow[t]{2}{*}{ Ru-H ( IRU) } & $1.51(2)$ & $\mathrm{C}(34)-\mathrm{H}(34)$ & 0.93 \\
\hline & & $C(35)-C(36)$ & $1.392(4)$ \\
\hline $\mathrm{Fe}-\mathrm{C}(11)$ & $1.999(2)$ & $\mathrm{C}(35)-\mathrm{H}(35)$ & 0.93 \\
\hline $\mathrm{Fe}-\mathrm{C}(21)$ & $2.002(2)$ & $\mathrm{C}(36)-\mathrm{H}(36)$ & 0.93 \\
\hline $\mathrm{Fe}-\mathrm{C}(12)$ & $2.020(3)$ & $C(41)-C(42)$ & $1.381(3)$ \\
\hline $\mathrm{Fe}-\mathrm{C}(25)$ & $2.023(2)$ & $C(41)-C(46)$ & $1.385(3)$ \\
\hline $\mathrm{Fe}-\mathrm{C}(15)$ & $2.030(2)$ & $C(42)-C(43)$ & $1.390(3)$ \\
\hline $\mathrm{Fe}-\mathrm{C}(22)$ & $2.040(2)$ & $\mathrm{C}(42)-\mathrm{H}(42)$ & 0.93 \\
\hline $\mathrm{Fe}-\mathrm{C}(24)$ & $2.046(2)$ & $C(43)-C(44)$ & $1.367(5)$ \\
\hline $\mathrm{Fe}-\mathrm{C}(23)$ & $2.048(2)$ & $\mathrm{C}(43)-\mathrm{H}(43)$ & 0.93 \\
\hline $\mathrm{Fe}-\mathrm{C}(13)$ & $2.053(3)$ & $C(44)-C(45)$ & $1.371(6)$ \\
\hline \multirow[t]{2}{*}{$\mathrm{Fe}-\mathrm{C}(14)$} & $2.056(3)$ & $\mathrm{C}(44)-\mathrm{H}(44)$ & 0.93 \\
\hline & & $C(45)-C(46)$ & $1.389(4)$ \\
\hline$P(1)-C(22)$ & $1.811(2)$ & $\mathrm{C}(45)-\mathrm{H}(45)$ & 0.93 \\
\hline$P(1)-C(41)$ & $1.839(2)$ & $\mathrm{C}(46)-\mathrm{H}(46)$ & 0.93 \\
\hline$P(1)-C(31)$ & $1.841(2)$ & $C(51)-C(52)$ & $1.386(3)$ \\
\hline$P(2)-C(51)$ & $1.837(2)$ & $C(51)-C(56)$ & $1.392(3)$ \\
\hline$P(2)-C(61)$ & $1.838(2)$ & $C(52)-C(53)$ & $1.391(3)$ \\
\hline \multirow[t]{2}{*}{$P(2)-C(1)$} & $1.862(2)$ & $\mathrm{C}(52)-\mathrm{H}(52)$ & 0.93 \\
\hline & & $C(53)-C(54)$ & $1.380(5)$ \\
\hline$C(1)-C(21)$ & $1.512(3)$ & $\mathrm{C}(53)-\mathrm{H}(53)$ & 0.93 \\
\hline$C(1)-C(2)$ & $1.550(3)$ & $C(54)-C(55)$ & $1.372(5)$ \\
\hline $\mathrm{C}(1)-\mathrm{H}(1)$ & 0.98 & $\mathrm{C}(54)-\mathrm{H}(54)$ & 0.93 \\
\hline$C(2)-C(3)$ & $1.526(3)$ & $C(55)-C(56)$ & $1.384(4)$ \\
\hline $\mathrm{C}(2)-\mathrm{H}(2 \mathrm{~A})$ & 0.97 & $\mathrm{C}(55)-\mathrm{H}(55)$ & 0.93 \\
\hline $\mathrm{C}(2)-\mathrm{H}(2 \mathrm{~B})$ & 0.97 & $\mathrm{C}(56)-\mathrm{H}(56)$ & 0.93 \\
\hline$C(3)-C(11)$ & $1.492(4)$ & $C(61)-C(62)$ & $1.387(3)$ \\
\hline $\mathrm{C}(3)-\mathrm{H}(3 \mathrm{~A})$ & 0.97 & $C(61)-C(66)$ & $1.391(3)$ \\
\hline $\mathrm{C}(3)-\mathrm{H}(3 \mathrm{~B})$ & 0.97 & $C(62)-C(63)$ & $1.380(4)$ \\
\hline$C(11)-C(12)$ & $1.423(4)$ & $\mathrm{C}(62)-\mathrm{H}(62)$ & 0.93 \\
\hline$C(11)-C(15)$ & $1.424(3)$ & $C(63)-C(64)$ & $1.371(5)$ \\
\hline$C(12)-C(13)$ & $1.428(4)$ & $\mathrm{C}(63)-\mathrm{H}(63)$ & 0.93 \\
\hline $\mathrm{C}(12)-\mathrm{H}(12)$ & 0.93 & $C(64)-C(65)$ & $1.376(5)$ \\
\hline$C(13)-C(14)$ & $1.408(4)$ & $\mathrm{C}(64)-\mathrm{H}(64)$ & 0.93 \\
\hline $\mathrm{C}(13)-\mathrm{H}(13)$ & 0.93 & $C(65)-C(66)$ & $1.382(4)$ \\
\hline$C(14)-C(15)$ & $1.412(4)$ & $\mathrm{C}(65)-\mathrm{H}(65)$ & 0.93 \\
\hline $\mathrm{C}(14)-\mathrm{H}(14)$ & 0.93 & $\mathrm{C}(66)-\mathrm{H}(66)$ & 0.93 \\
\hline $\mathrm{C}(15)-\mathrm{H}(15)$ & 0.93 & $C(71)-C(75)$ & $1.390(5)$ \\
\hline$C(21)-C(25)$ & $1.434(3)$ & $C(71)-C(72)$ & $1.400(5)$ \\
\hline$C(21)-C(22)$ & $1.450(3)$ & $\mathrm{C}(71)-\mathrm{H}(71)$ & 0.93 \\
\hline$C(22)-C(23)$ & $1.433(3)$ & $C(72)-C(73)$ & $1.403(5)$ \\
\hline$C(23)-C(24)$ & $1.414(3)$ & $\mathrm{C}(72)-\mathrm{H}(72)$ & 0.93 \\
\hline $\mathrm{C}(23)-\mathrm{H}(23)$ & 0.93 & $C(73)-C(74)$ & $1.387(5)$ \\
\hline$C(24)-C(25)$ & $1.413(4)$ & $\mathrm{C}(73)-\mathrm{H}(73)$ & 0.93 \\
\hline
\end{tabular}




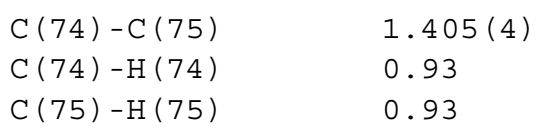

\author{
$1.405(4)$ \\ 0.93
}

0.93

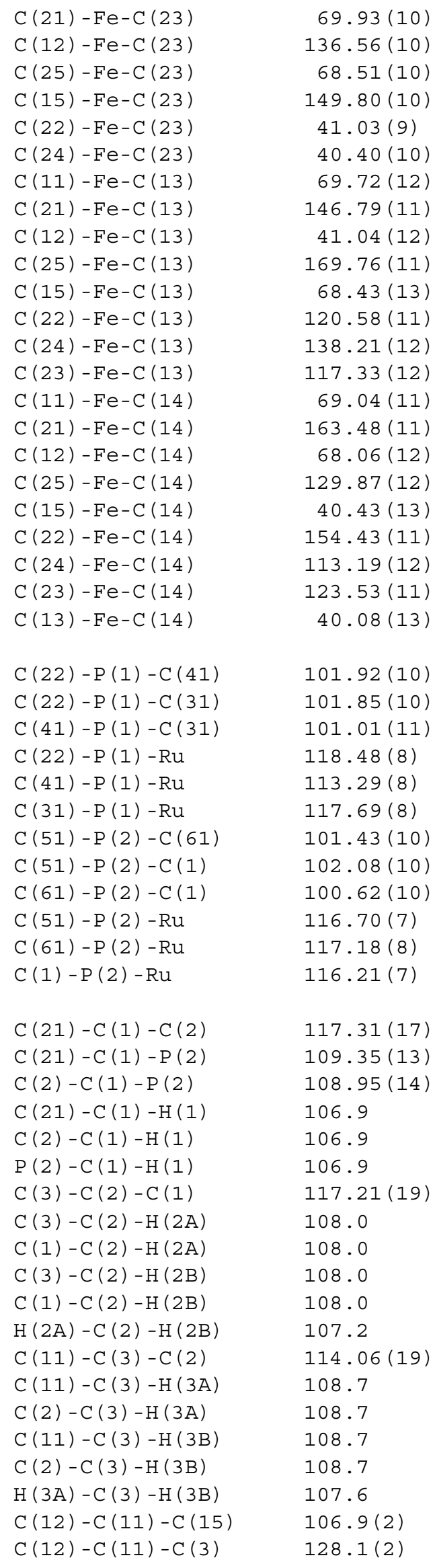


S8

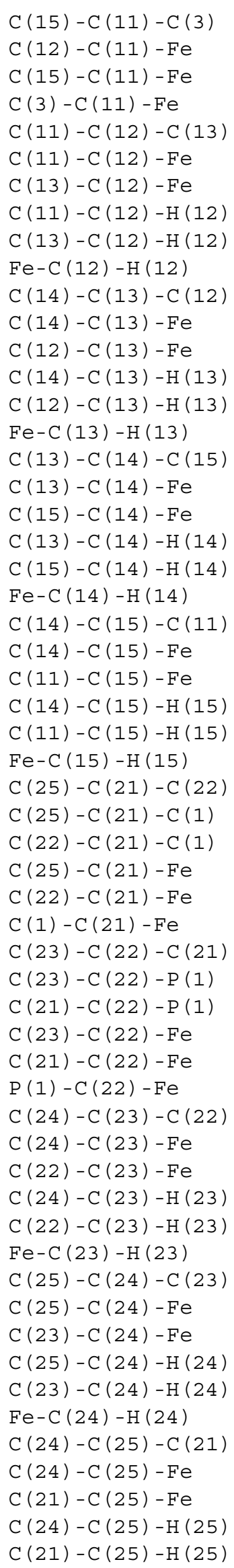

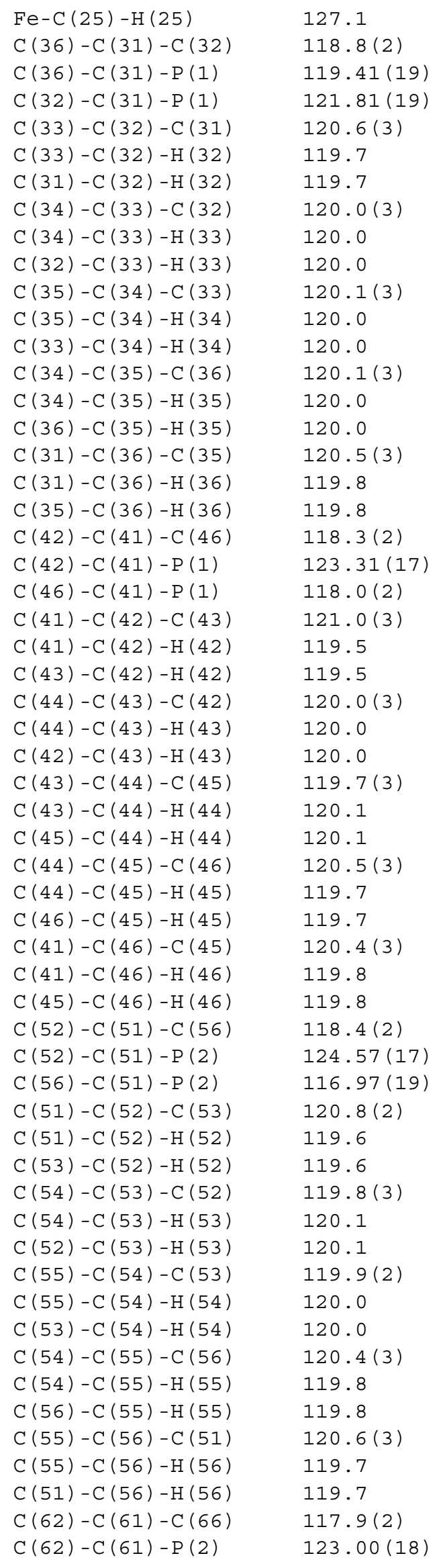




$\begin{array}{ll}\mathrm{C}(66)-\mathrm{C}(61)-\mathrm{P}(2) & 119.09(17) \\ \mathrm{C}(63)-\mathrm{C}(62)-\mathrm{C}(61) & 120.9(3) \\ \mathrm{C}(63)-\mathrm{C}(62)-\mathrm{H}(62) & 119.6 \\ \mathrm{C}(61)-\mathrm{C}(62)-\mathrm{H}(62) & 119.6 \\ \mathrm{C}(64)-\mathrm{C}(63)-\mathrm{C}(62) & 120.6(3) \\ \mathrm{C}(64)-\mathrm{C}(63)-\mathrm{H}(63) & 119.7 \\ \mathrm{C}(62)-\mathrm{C}(63)-\mathrm{H}(63) & 119.7 \\ \mathrm{C}(63)-\mathrm{C}(64)-\mathrm{C}(65) & 119.4(3) \\ \mathrm{C}(63)-\mathrm{C}(64)-\mathrm{H}(64) & 120.3 \\ \mathrm{C}(65)-\mathrm{C}(64)-\mathrm{H}(64) & 120.3 \\ \mathrm{C}(64)-\mathrm{C}(65)-\mathrm{C}(66) & 120.3(3) \\ \mathrm{C}(64)-\mathrm{C}(65)-\mathrm{H}(65) & 119.9 \\ \mathrm{C}(66)-\mathrm{C}(65)-\mathrm{H}(65) & 119.9 \\ \mathrm{C}(65)-\mathrm{C}(66)-\mathrm{C}(61) & 120.9(2) \\ \mathrm{C}(65)-\mathrm{C}(66)-\mathrm{H}(66) & 119.5 \\ \mathrm{C}(61)-\mathrm{C}(66)-\mathrm{H}(66) & 119.5 \\ \mathrm{C}(75)-\mathrm{C}(71)-\mathrm{C}(72) & 108.2(3) \\ \mathrm{C}(75)-\mathrm{C}(71)-\mathrm{Ru} & 72.38(16) \\ \mathrm{C}(72)-\mathrm{C}(71)-\mathrm{Ru} & 72.28(16) \\ \mathrm{C}(75)-\mathrm{C}(71)-\mathrm{H}(71) & 125.9 \\ \mathrm{C}(72)-\mathrm{C}(71)-\mathrm{H}(71) & 125.9 \\ \mathrm{Ru}-\mathrm{C}(71)-\mathrm{H}(71) & 121.2 \\ \mathrm{C}(71)-\mathrm{C}(72)-\mathrm{C}(73) & 107.4(3) \\ & \end{array}$

$\begin{array}{lc}C(71)-\mathrm{C}(72)-\mathrm{Ru} & 71.15(17) \\ \mathrm{C}(73)-\mathrm{C}(72)-\mathrm{Ru} & 72.59(16) \\ \mathrm{C}(71)-\mathrm{C}(72)-\mathrm{H}(72) & 126.3 \\ \mathrm{C}(73)-\mathrm{C}(72)-\mathrm{H}(72) & 126.3 \\ \mathrm{Ru}-\mathrm{C}(72)-\mathrm{H}(72) & 121.7 \\ \mathrm{C}(74)-\mathrm{C}(73)-\mathrm{C}(72) & 108.5(3) \\ \mathrm{C}(74)-\mathrm{C}(73)-\mathrm{Ru} & 72.76(16) \\ \mathrm{C}(72)-\mathrm{C}(73)-\mathrm{Ru} & 71.06(17) \\ \mathrm{C}(74)-\mathrm{C}(73)-\mathrm{H}(73) & 125.8 \\ \mathrm{C}(72)-\mathrm{C}(73)-\mathrm{H}(73) & 125.8 \\ \mathrm{Ru}-\mathrm{C}(73)-\mathrm{H}(73) & 122.1 \\ \mathrm{C}(73)-\mathrm{C}(74)-\mathrm{C}(75) & 107.8(3) \\ \mathrm{C}(73)-\mathrm{C}(74)-\mathrm{Ru} & 71.61(16) \\ \mathrm{C}(75)-\mathrm{C}(74)-\mathrm{Ru} & 70.49(15) \\ \mathrm{C}(73)-\mathrm{C}(74)-\mathrm{H}(74) & 126.1 \\ \mathrm{C}(75)-\mathrm{C}(74)-\mathrm{H}(74) & 126.1 \\ \mathrm{Ru}-\mathrm{C}(74)-\mathrm{H}(74) & 123.4 \\ \mathrm{C}(71)-\mathrm{C}(75)-\mathrm{C}(74) & 108.1(3) \\ \mathrm{C}(71)-\mathrm{C}(75)-\mathrm{Ru} & 71.33(16) \\ \mathrm{C}(74)-\mathrm{C}(75)-\mathrm{Ru} & 73.24(15) \\ \mathrm{C}(71)-\mathrm{C}(75)-\mathrm{H}(75) & 126.0 \\ \mathrm{C}(74)-\mathrm{C}(75)-\mathrm{H}(75) & 126.0 \\ \mathrm{Ru}-\mathrm{C}(75)-\mathrm{H}(75) & 121.2\end{array}$


Figure. S1. Thermal ellipsoid plot (20\% ellipsoids) of $\mathrm{CpRuH}\left(\mathrm{PP}^{\mathrm{Ph}} \mathrm{PF}\right),(4)$.

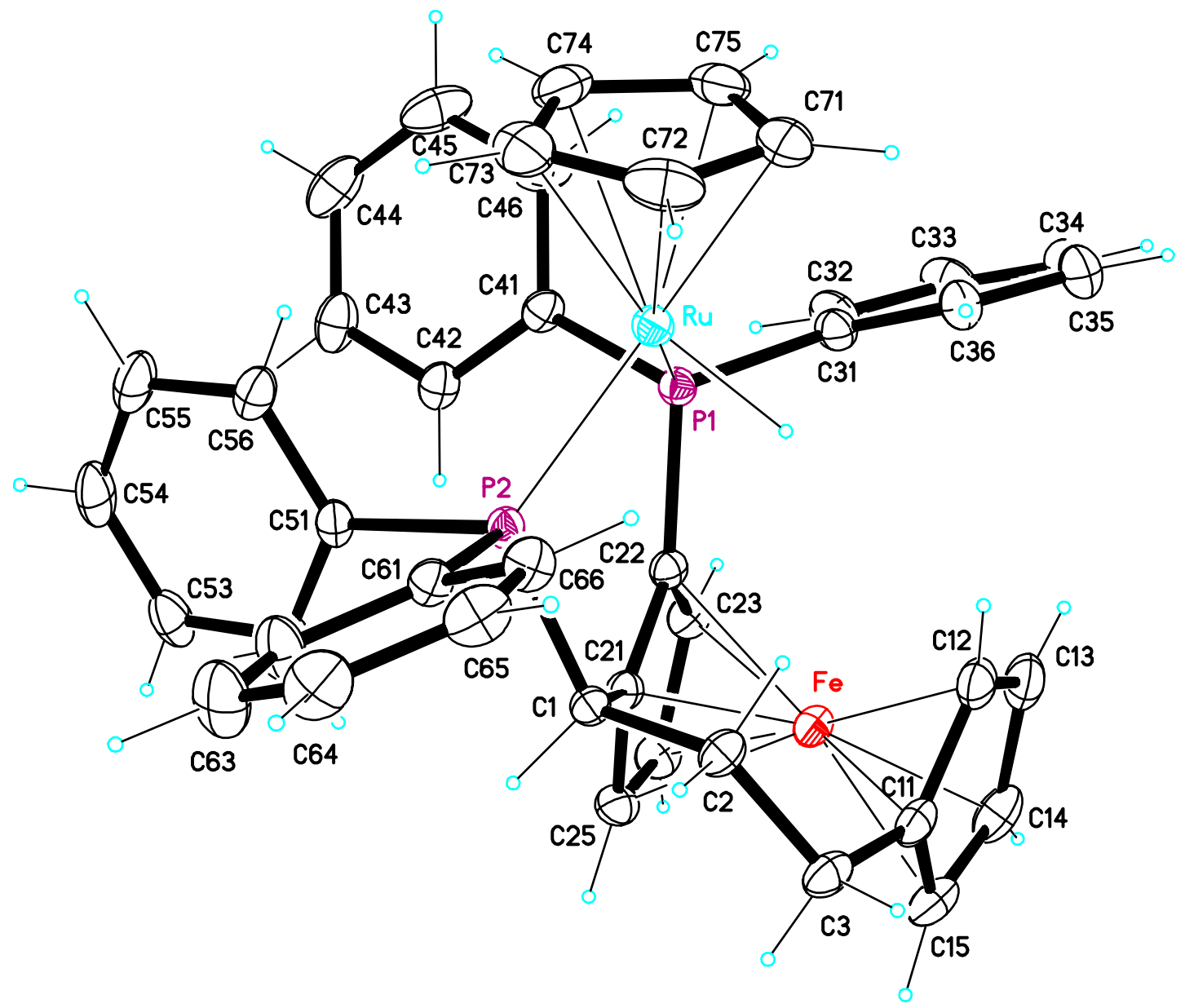


Table S6. Crystal data and structure refinement for $\mathrm{CpRu}\left(\mathrm{CF}_{3} \mathrm{CO}_{2}\right)\left(\mathrm{PP}^{\mathrm{Ph}} \mathrm{PF}\right),(\mathbf{1 3})$.

Identification code
Empirical formula
Formula weight
Temperature
Wavelength
Crystal system, space group
Unit cell dimensions

Volume

Z, Calculated density

Absorption coefficient

$\mathrm{F}(000)$

Crystal size

Diffractometer

Scan type / width / speed

Theta range for data collection

Index ranges

Reflections collected / unique

Completeness to theta $=30.00$

Absorption correction

Transmission factors

Structure solution

Refinement method

Data / restraints / parameters

Goodness-of-fit on $\mathrm{F}^{2}$

Final $\mathrm{R}$ indices [I $>2 \operatorname{sigma}(\mathrm{I})]$

$\mathrm{R}$ indices (all data)

Largest diff. peak and hole 512wein

C44 H37 F3 Fe O2 P2 Ru

873.60

297(2) K

$0.71073 \AA$

Triclinic, P-1 (no. 2)

$\mathrm{a}=11.350(5) \AA \quad \alpha=82.07(2) \mathrm{deg}$.

$\mathrm{b}=11.543(5) \AA \quad \beta=88.66(2) \mathrm{deg}$.

$\mathrm{c}=16.084(7) \AA \quad \gamma=62.05(2) \mathrm{deg}$.

$1841.6(14) \AA^{3}$

2, $1.575 \mathrm{Mg} / \mathrm{m}^{3}$

$0.945 \mathrm{~mm}^{-1}$

888

$0.14 \times 0.24$ x $0.64 \mathrm{~mm}$, orange plate

Bruker SMART CCD platform type 3-circle

(sealed X-ray tube, Mo K $\alpha$ radiation, graphite

monochr., det.dist. $44.50 \mathrm{~mm}, 512 \times 512$ pixels)

$\omega$-scan frames $/ \Delta \omega=0.3^{\circ} / 20 \mathrm{sec}$. per frame

full sphere data collection, 4 x 606 frames

2.02 to $30.00 \mathrm{deg}$.

$-15<=\mathrm{h}<=15,-15<=\mathrm{k}<=16,-22<=\mathrm{l}<=22$

$33751 / 10525[\mathrm{R}($ int $)=0.046, \mathrm{R}($ sigma $)=0.035]$

$98.1 \%$

Multi-scan (program SADABS; Sheldrick, 1996)

$0.86-0.58$

Direct methods (program SHELXS97)

Full-matrix least-squares on $\mathrm{F}^{2}$ (prg SHELXL97) 10525 / 0 / 478

1.016

$\mathrm{R} 1=0.0328, \mathrm{wR} 2=0.0830 \quad(8557$ data $)$

$\mathrm{R} 1=0.0449, \mathrm{wR} 2=0.0901 \quad(10525$ data $)$

0.65 and -0.57 e $\AA^{-3}$

$\mathrm{R} 1=\Sigma|| \mathrm{F}_{\mathrm{o}}|-| \mathrm{F}_{\mathrm{c}}|/ \Sigma| \mathrm{F}_{\mathrm{o}} \mid, \mathrm{wR} 2=\left[\Sigma\left(\mathrm{w}\left(\mathrm{F}_{\mathrm{o}}{ }^{2}-\mathrm{F}_{\mathrm{c}}{ }^{2}\right)^{2}\right) / \Sigma\left(\mathrm{w}\left(\mathrm{F}_{\mathrm{o}}{ }^{2}\right)^{2}\right)\right]^{1 / 2}$ 


\section{S12}

Table S7. Atomic coordinates and equivalent isotropic displacement parameters $\left(\AA^{2} \times 10^{3}\right)$ for $\mathrm{CpRu}\left(\mathrm{CF}_{3} \mathrm{CO}_{2}\right)\left(\mathrm{PP}^{\mathrm{Ph}} \mathrm{PF}\right),(\mathbf{1 3}) . \mathrm{U}_{\text {eq }}$ is defined as one third of the trace of the orthogonalized $\mathrm{U}_{\mathrm{ij}}$ tensor.

\begin{tabular}{|c|c|c|c|c|}
\hline & $x$ & $\mathrm{y}$ & $z$ & $\mathrm{U}_{\mathrm{eq}}$ \\
\hline $\mathrm{Ru}$ & $0.12019(1)$ & $0.42418(1)$ & $0.25945(1)$ & $32(1)$ \\
\hline $\mathrm{Fe}$ & $0.59308(3)$ & $0.04807(3)$ & $0.19019(2)$ & $38(1)$ \\
\hline $\mathrm{P}(1)$ & $0.24429(5)$ & $0.28202(4)$ & $0.16351(3)$ & $31(1)$ \\
\hline $\mathrm{P}(2)$ & $0.27733(5)$ & $0.29793(5)$ & $0.36710(3)$ & $33(1)$ \\
\hline$C(1)$ & $0.4537(2)$ & $0.2018(2)$ & $0.3376(1)$ & $35(1)$ \\
\hline$C(2)$ & $0.5020(2)$ & $0.3005(2)$ & $0.2965(1)$ & $41(1)$ \\
\hline$C(3)$ & $0.6509(2)$ & $0.2364(2)$ & $0.2784(2)$ & $47(1)$ \\
\hline$C(11)$ & $0.6890(2)$ & $0.1531(2)$ & $0.2088(2)$ & $45(1)$ \\
\hline$C(12)$ & $0.6288(2)$ & $0.1931(2)$ & $0.1255(1)$ & $45(1)$ \\
\hline$C(13)$ & $0.6822(2)$ & $0.0810(3)$ & $0.0826(2)$ & $55(1)$ \\
\hline$C(14)$ & $0.7767(2)$ & $-0.0286(3)$ & $0.1381(2)$ & $61(1)$ \\
\hline$C(15)$ & $0.7821(2)$ & $0.0155(2)$ & $0.2147(2)$ & $54(1)$ \\
\hline$C(21)$ & $0.4647(2)$ & $0.1017(2)$ & $0.2830(1)$ & $33(1)$ \\
\hline$C(22)$ & $0.3913(2)$ & $0.1276(2)$ & $0.2042(1)$ & $33(1)$ \\
\hline$C(23)$ & $0.4425(2)$ & $0.0016(2)$ & $0.1732(1)$ & $42(1)$ \\
\hline$C(24)$ & $0.5419(2)$ & $-0.0977(2)$ & $0.2319(2)$ & $48(1)$ \\
\hline$C(25)$ & $0.5549(2)$ & $-0.0373(2)$ & $0.2990(1)$ & $42(1)$ \\
\hline$C(31)$ & $0.2985(2)$ & $0.3465(2)$ & $0.0677(1)$ & $35(1)$ \\
\hline$C(32)$ & $0.3623(2)$ & $0.2684(2)$ & $0.0053(1)$ & $46(1)$ \\
\hline$C(33)$ & $0.4049(3)$ & $0.3192(3)$ & $-0.0650(1)$ & $54(1)$ \\
\hline$C(34)$ & $0.3847(3)$ & $0.4480(3)$ & $-0.0749(2)$ & $59(1)$ \\
\hline$C(35)$ & $0.3211(3)$ & $0.5261(3)$ & $-0.0142(2)$ & $65(1)$ \\
\hline$C(36)$ & $0.2789(3)$ & $0.4752(2)$ & $0.0573(1)$ & $49(1)$ \\
\hline$C(41)$ & $0.1452(2)$ & $0.2156(2)$ & $0.1177(1)$ & $42(1)$ \\
\hline$C(42)$ & $0.1247(2)$ & $0.1176(2)$ & $0.1660(2)$ & $60(1)$ \\
\hline$C(43)$ & $0.0412(3)$ & $0.0732(3)$ & $0.1367(3)$ & $79(1)$ \\
\hline$C(44)$ & $-0.0215(3)$ & $0.1265(3)$ & $0.0577(3)$ & $81(1)$ \\
\hline$C(45)$ & $-0.0012(3)$ & $0.2226(4)$ & $0.0090(2)$ & $73(1)$ \\
\hline$C(46)$ & $0.0812(2)$ & $0.2686(3)$ & $0.0387(2)$ & $53(1)$ \\
\hline$C(51)$ & $0.2367(2)$ & $0.1776(2)$ & $0.4322(1)$ & $39(1)$ \\
\hline$C(52)$ & $0.2596(3)$ & $0.0591(2)$ & $0.4065(2)$ & $54(1)$ \\
\hline$C(53)$ & $0.2149(3)$ & $-0.0226(3)$ & $0.4525(2)$ & $67(1)$ \\
\hline$C(54)$ & $0.1442(3)$ & $0.0145(3)$ & $0.5239(2)$ & $62(1)$ \\
\hline$C(55)$ & $0.1182(3)$ & $0.1327(3)$ & $0.5493(2)$ & $62(1)$ \\
\hline$C(56)$ & $0.1658(3)$ & $0.2132(3)$ & $0.5045(1)$ & $52(1)$ \\
\hline$C(61)$ & $0.3018(2)$ & $0.3835(2)$ & $0.4482(1)$ & $41(1)$ \\
\hline$C(62)$ & $0.3978(3)$ & $0.3128(3)$ & $0.5136(2)$ & $58(1)$ \\
\hline$C(63)$ & $0.4192(3)$ & $0.3772(4)$ & $0.5733(2)$ & $74(1)$ \\
\hline$C(64)$ & $0.3460(3)$ & $0.5128(4)$ & $0.5677(2)$ & $76(1)$ \\
\hline$C(65)$ & $0.2496(3)$ & $0.5843(3)$ & $0.5048(2)$ & $66(1)$ \\
\hline$C(66)$ & $0.2259(3)$ & $0.5204(2)$ & $0.4444(2)$ & $49(1)$ \\
\hline$C(71)$ & $-0.0859(2)$ & $0.5734(3)$ & $0.2123(2)$ & $69(1)$ \\
\hline$C(72)$ & $-0.0717(2)$ & $0.5819(3)$ & $0.2968(2)$ & $67(1)$ \\
\hline$C(73)$ & $-0.0459(3)$ & $0.4660(4)$ & $0.3429(2)$ & $80(1)$ \\
\hline$C(74)$ & $-0.0396(3)$ & $0.3781(3)$ & $0.2899(4)$ & $95(1)$ \\
\hline$C(75)$ & $-0.0683(3)$ & $0.4484(4)$ & $0.2072(3)$ & $88(1)$ \\
\hline$O(1)$ & $0.2250(2)$ & $0.5424(2)$ & $0.2389(1)$ & $43(1)$ \\
\hline$O(2)$ & $0.0725(2)$ & $0.7459(2)$ & $0.2603(2)$ & $107(1)$ \\
\hline$C(4)$ & $0.1822(2)$ & $0.6614(2)$ & $0.2435(2)$ & $49(1)$ \\
\hline
\end{tabular}




\section{S13}

\begin{tabular}{llllr}
$C(5)$ & $0.2890(3)$ & $0.7080(2)$ & $0.2283(2)$ & $55(1)$ \\
$F(1)$ & $0.2840(2)$ & $0.7571(2)$ & $0.1476(1)$ & $104(1)$ \\
$F(2)$ & $0.4139(2)$ & $0.6133(2)$ & $0.2464(1)$ & $83(1)$ \\
$F(3)$ & $0.2710(2)$ & $0.8040(2)$ & $0.2709(2)$ & $105(1)$ \\
\hline
\end{tabular}

Table S8. Hydrogen coordinates and isotropic displacement parameters $\left(\AA^{2} \times 10^{3}\right)$ for $\mathrm{CpRu}\left(\mathrm{CF}_{3} \mathrm{CO}_{2}\right)\left(\mathrm{PP}^{\mathrm{Ph}} \mathrm{PF}\right)$, (13).

\begin{tabular}{|c|c|c|c|c|}
\hline & $x$ & $\mathrm{Y}$ & $z$ & $U_{\text {eq }}$ \\
\hline $\mathrm{H}(1)$ & 0.5086 & 0.1537 & 0.3893 & 42 \\
\hline $\mathrm{H}(2 \mathrm{~A})$ & 0.4506 & 0.3462 & 0.2441 & 49 \\
\hline $\mathrm{H}(2 \mathrm{~B})$ & 0.4838 & 0.3664 & 0.3332 & 49 \\
\hline $\mathrm{H}(3 \mathrm{~A})$ & 0.6763 & 0.3059 & 0.2643 & 57 \\
\hline $\mathrm{H}(3 \mathrm{~B})$ & 0.7018 & 0.1815 & 0.3293 & 57 \\
\hline $\mathrm{H}(12)$ & 0.5653 & 0.2784 & 0.1033 & 54 \\
\hline $\mathrm{H}(13)$ & 0.6593 & 0.0796 & 0.0278 & 66 \\
\hline $\mathrm{H}(14)$ & 0.8264 & -0.1147 & 0.1261 & 73 \\
\hline $\mathrm{H}(15)$ & 0.8374 & -0.0365 & 0.2615 & 65 \\
\hline $\mathrm{H}(23)$ & 0.4148 & -0.0120 & 0.1231 & 50 \\
\hline $\mathrm{H}(24)$ & 0.5902 & -0.1873 & 0.2269 & 58 \\
\hline $\mathrm{H}(25)$ & 0.6127 & -0.0807 & 0.3461 & 51 \\
\hline $\mathrm{H}(32)$ & 0.3763 & 0.1816 & 0.0111 & 55 \\
\hline $\mathrm{H}(33)$ & 0.4475 & 0.2661 & -0.1060 & 64 \\
\hline $\mathrm{H}(34)$ & 0.4139 & 0.4816 & -0.1221 & 71 \\
\hline $\mathrm{H}(35)$ & 0.3060 & 0.6133 & -0.0207 & 78 \\
\hline $\mathrm{H}(36)$ & 0.2370 & 0.5285 & 0.0983 & 59 \\
\hline $\mathrm{H}(42)$ & 0.1678 & 0.0812 & 0.2188 & 72 \\
\hline $\mathrm{H}(43)$ & 0.0275 & 0.0083 & 0.1698 & 95 \\
\hline $\mathrm{H}(44)$ & -0.0775 & 0.0972 & 0.0375 & 98 \\
\hline $\mathrm{H}(45)$ & -0.0429 & 0.2574 & -0.0442 & 87 \\
\hline $\mathrm{H}(46)$ & 0.0934 & 0.3346 & 0.0057 & 64 \\
\hline $\mathrm{H}(52)$ & 0.3054 & 0.0341 & 0.3579 & 64 \\
\hline $\mathrm{H}(53)$ & 0.2326 & -0.1027 & 0.4353 & 81 \\
\hline $\mathrm{H}(54)$ & 0.1143 & -0.0404 & 0.5545 & 75 \\
\hline $\mathrm{H}(55)$ & 0.0688 & 0.1590 & 0.5965 & 74 \\
\hline $\mathrm{H}(56 \mathrm{~A})$ & 0.1500 & 0.2918 & 0.5231 & 62 \\
\hline $\mathrm{H}(62 \mathrm{~A})$ & 0.4480 & 0.2213 & 0.5171 & 69 \\
\hline $\mathrm{H}(63 \mathrm{~A})$ & 0.4828 & 0.3291 & 0.6171 & 88 \\
\hline $\mathrm{H}(64)$ & 0.3621 & 0.5564 & 0.6071 & 91 \\
\hline $\mathrm{H}(65)$ & 0.1997 & 0.6757 & 0.5022 & 79 \\
\hline $\mathrm{H}(66)$ & 0.1600 & 0.5689 & 0.4019 & 59 \\
\hline $\mathrm{H}(71)$ & -0.1041 & 0.6404 & 0.1674 & 83 \\
\hline $\mathrm{H}(72)$ & -0.0790 & 0.6560 & 0.3178 & 80 \\
\hline $\mathrm{H}(73)$ & -0.0342 & 0.4474 & 0.4011 & 96 \\
\hline $\mathrm{H}(74)$ & -0.0202 & 0.2902 & 0.3056 & 114 \\
\hline $\mathrm{H}(75)$ & -0.0741 & 0.4160 & 0.1584 & 106 \\
\hline
\end{tabular}

Hydrogen atoms inserted in idealized positions and refined riding with the atoms to which they were bonded. All $\mathrm{H}$ atoms had $\mathrm{U}_{\mathrm{iso}}=\mathrm{U}_{\mathrm{eq}} \times 1.2\left(\mathrm{x} 1.5\right.$ for $\left.\mathrm{CH}_{3}\right)$ of their carrier atoms. 


\section{S14}

Table S9. Anisotropic displacement parameters $\left(\AA^{2} \times 10^{3}\right)$ for $\mathrm{CpRu}\left(\mathrm{CF}_{3} \mathrm{CO}_{2}\right)\left(\mathrm{PP}^{\mathrm{Ph}} \mathrm{PF}\right),(\mathbf{1 3})$.

The anisotropic displacement factor exponent takes the form: $-2 \pi^{2}\left[h^{2} a^{* 2} U_{11}+\ldots\right.$ $+2 \mathrm{~h} \mathrm{k} \mathrm{a}^{*} \mathrm{~b}^{*} \mathrm{U}_{12}$ ].

\begin{tabular}{|c|c|c|c|c|c|c|}
\hline & $\mathrm{U}_{11}$ & $\mathrm{U}_{22}$ & $\mathrm{U}_{33}$ & $\mathrm{U}_{23}$ & $\mathrm{U}_{13}$ & $\mathrm{U}_{12}$ \\
\hline $\mathrm{Ru}$ & $32(1)$ & $33(1)$ & $33(1)$ & $-5(1)$ & $2(1)$ & $-15(1)$ \\
\hline $\mathrm{Fe}$ & $35(1)$ & $34(1)$ & $40(1)$ & $-5(1)$ & $4(1)$ & $-12(1)$ \\
\hline $\mathrm{P}(1)$ & $33(1)$ & $30(1)$ & $31(1)$ & $-5(1)$ & $-1(1)$ & $-17(1)$ \\
\hline$P(2)$ & $37(1)$ & $36(1)$ & $29(1)$ & $-6(1)$ & $1(1)$ & $-19(1)$ \\
\hline$C(1)$ & $36(1)$ & $38(1)$ & $31(1)$ & $-3(1)$ & $-3(1)$ & $-19(1)$ \\
\hline$C(2)$ & $41(1)$ & 41 (1) & $46(1)$ & $-8(1)$ & $1(1)$ & $-23(1)$ \\
\hline$C(3)$ & $40(1)$ & $56(1)$ & $55(1)$ & $-9(1)$ & $1(1)$ & $-29(1)$ \\
\hline$C(11)$ & $33(1)$ & $50(1)$ & $51(1)$ & $-4(1)$ & $3(1)$ & $-21(1)$ \\
\hline$C(12)$ & $40(1)$ & $50(1)$ & $47(1)$ & $0(1)$ & $4(1)$ & $-23(1)$ \\
\hline$C(13)$ & $51(1)$ & $69(2)$ & $46(1)$ & $-11(1)$ & $14(1)$ & $-28(1)$ \\
\hline$C(14)$ & $44(1)$ & $59(1)$ & $70(2)$ & $-18(1)$ & $19(1)$ & $-14(1)$ \\
\hline$C(15)$ & $32(1)$ & $55(1)$ & $60(1)$ & $0(1)$ & $2(1)$ & $-11(1)$ \\
\hline$C(21)$ & $36(1)$ & $30(1)$ & $33(1)$ & $-2(1)$ & $2(1)$ & $-16(1)$ \\
\hline$C(22)$ & $35(1)$ & 31 (1) & $35(1)$ & $-6(1)$ & $2(1)$ & $-17(1)$ \\
\hline$C(23)$ & 49 (1) & $33(1)$ & $46(1)$ & $-11(1)$ & $2(1)$ & $-19(1)$ \\
\hline$C(24)$ & $52(1)$ & $29(1)$ & $58(1)$ & $-5(1)$ & $2(1)$ & $-15(1)$ \\
\hline$C(25)$ & $43(1)$ & $34(1)$ & $44(1)$ & $1(1)$ & $-1(1)$ & $-15(1)$ \\
\hline$C(31)$ & $37(1)$ & 39 (1) & $29(1)$ & $-3(1)$ & $0(1)$ & $-19(1)$ \\
\hline$C(32)$ & $55(1)$ & $46(1)$ & 39 (1) & $-11(1)$ & $5(1)$ & $-25(1)$ \\
\hline C (33) & $60(1)$ & $66(2)$ & 36 (1) & $-13(1)$ & $9(1)$ & $-28(1)$ \\
\hline$C(34)$ & $68(2)$ & $68(2)$ & $40(1)$ & $1(1)$ & $14(1)$ & $-33(1)$ \\
\hline$C(35)$ & $88(2)$ & $51(1)$ & $59(2)$ & $-4(1)$ & $25(1)$ & $-37(1)$ \\
\hline$C(36)$ & $66(1)$ & 41 (1) & $43(1)$ & $-7(1)$ & $13(1)$ & $-27(1)$ \\
\hline$C(41)$ & $35(1)$ & $42(1)$ & $53(1)$ & $-17(1)$ & $1(1)$ & $-20(1)$ \\
\hline$C(42)$ & $48(1)$ & 49 (1) & $91(2)$ & $-9(1)$ & $-2(1)$ & $-31(1)$ \\
\hline$C(43)$ & $50(1)$ & $60(2)$ & $145(3)$ & $-28(2)$ & $6(2)$ & $-36(1)$ \\
\hline$C(44)$ & $46(1)$ & $87(2)$ & $134(3)$ & $-62(2)$ & $11(2)$ & $-39(2)$ \\
\hline$C(45)$ & $42(1)$ & $108(2)$ & $77(2)$ & $-53(2)$ & $1(1)$ & $-31(2)$ \\
\hline$C(46)$ & $42(1)$ & $71(2)$ & $50(1)$ & $-24(1)$ & $1(1)$ & $-26(1)$ \\
\hline$C(51)$ & $42(1)$ & $45(1)$ & $35(1)$ & $0(1)$ & $-2(1)$ & $-25(1)$ \\
\hline$C(52)$ & $62(1)$ & $52(1)$ & $58(1)$ & $-9(1)$ & $14(1)$ & $-36(1)$ \\
\hline$C(53)$ & $76(2)$ & $60(2)$ & $80(2)$ & $-5(1)$ & $10(2)$ & $-46(2)$ \\
\hline$C(54)$ & $63(2)$ & $75(2)$ & $57(2)$ & $17(1)$ & $-5(1)$ & $-45(1)$ \\
\hline$C(55)$ & $65(2)$ & $88(2)$ & $38(1)$ & $3(1)$ & $5(1)$ & $-44(2)$ \\
\hline$C(56)$ & $63(1)$ & $62(1)$ & $36(1)$ & $-6(1)$ & $6(1)$ & $-35(1)$ \\
\hline$C(61)$ & 49 (1) & $51(1)$ & $33(1)$ & $-12(1)$ & $4(1)$ & $-30(1)$ \\
\hline$C(62)$ & $58(1)$ & $70(2)$ & $47(1)$ & $-16(1)$ & $-6(1)$ & $-30(1)$ \\
\hline$C(63)$ & $72(2)$ & $110(3)$ & $52(2)$ & $-29(2)$ & $-5(1)$ & $-49(2)$ \\
\hline$C(64)$ & $85(2)$ & $115(3)$ & $66(2)$ & $-50(2)$ & $17(2)$ & $-70(2)$ \\
\hline$C(65)$ & $86(2)$ & $72(2)$ & $66(2)$ & $-38(1)$ & $26(2)$ & $-53(2)$ \\
\hline$C(66)$ & $60(1)$ & $53(1)$ & $44(1)$ & $-18(1)$ & $15(1)$ & $-32(1)$ \\
\hline$C(71)$ & $36(1)$ & $76(2)$ & $64(2)$ & $8(1)$ & $-1(1)$ & $-5(1)$ \\
\hline$C(72)$ & $39(1)$ & $70(2)$ & $91(2)$ & $-40(2)$ & $17(1)$ & $-18(1)$ \\
\hline$C(73)$ & $42(1)$ & $121(3)$ & $58(2)$ & $9(2)$ & $13(1)$ & $-30(2)$ \\
\hline$C(74)$ & $37(1)$ & $54(2)$ & $191(5)$ & $0(2)$ & $19(2)$ & $-24(1)$ \\
\hline$C(75)$ & $33(1)$ & $127(3)$ & $113(3)$ & $-78(3)$ & $9(1)$ & $-28(2)$ \\
\hline$O(1)$ & $48(1)$ & $44(1)$ & $43(1)$ & $-12(1)$ & $6(1)$ & $-26(1)$ \\
\hline$O(2)$ & $59(1)$ & $44(1)$ & $207(3)$ & $-19(2)$ & $28(2)$ & $-15(1)$ \\
\hline$C(4)$ & $47(1)$ & $38(1)$ & $57(1)$ & $-1(1)$ & $-1(1)$ & $-17(1)$ \\
\hline
\end{tabular}




\begin{tabular}{|c|c|c|c|c|c|c|}
\hline$C(5)$ & $61(1)$ & $40(1)$ & $68(2)$ & $-7(1)$ & $1(1)$ & $-27(1)$ \\
\hline$F(1)$ & $116(2)$ & $114(2)$ & $86(1)$ & $20(1)$ & $15(1)$ & $-66(2)$ \\
\hline$F(2)$ & $56(1)$ & $63(1)$ & $135(2)$ & $-7(1)$ & $-6(1)$ & $-33(1)$ \\
\hline$F(3)$ & $114(2)$ & 79 (1) & $150(2)$ & $-55(1)$ & $8(1)$ & $-59(1)$ \\
\hline
\end{tabular}

Table 10. Bond lengths $[\AA]$ and angles $[\mathrm{deg}]$ for $\mathrm{CpRu}\left(\mathrm{CF}_{3} \mathrm{CO}_{2}\right)\left(\mathrm{PP}^{\mathrm{Ph}} \mathrm{PF}\right)$, (13).

\begin{tabular}{|c|c|c|c|}
\hline \multicolumn{2}{|c|}{ Bond distances } & $C(13)-C(14)$ & $1.417(4)$ \\
\hline & & $\mathrm{C}(13)-\mathrm{H}(13)$ & 0.93 \\
\hline $\mathrm{Ru}-\mathrm{C}(74)$ & $2.145(3)$ & $C(14)-C(15)$ & $1.410(4)$ \\
\hline $\mathrm{Ru}-\mathrm{O}(1)$ & $2.183(2)$ & $\mathrm{C}(14)-\mathrm{H}(14)$ & 0.93 \\
\hline $\mathrm{Ru}-\mathrm{C}(73)$ & $2.190(3)$ & $\mathrm{C}(15)-\mathrm{H}(15)$ & 0.93 \\
\hline $\mathrm{Ru}-\mathrm{C}(75)$ & $2.195(3)$ & $C(21)-C(25)$ & $1.431(3)$ \\
\hline $\mathrm{Ru}-\mathrm{C}(72)$ & $2.223(2)$ & $C(21)-C(22)$ & $1.448(3)$ \\
\hline $\mathrm{Ru}-\mathrm{C}(71)$ & $2.227(3)$ & $C(22)-C(23)$ & $1.448(3)$ \\
\hline $\mathrm{Ru}-\mathrm{P}(2)$ & $2.305(1)$ & $C(23)-C(24)$ & $1.421(3)$ \\
\hline \multirow[t]{2}{*}{$R u-P(1)$} & $2.343(1)$ & $\mathrm{C}(23)-\mathrm{H}(23)$ & 0.93 \\
\hline & & $C(24)-C(25)$ & $1.410(3)$ \\
\hline $\mathrm{Fe}-\mathrm{C}(21)$ & $2.011(2)$ & $\mathrm{C}(24)-\mathrm{H}(24)$ & 0.93 \\
\hline $\mathrm{Fe}-\mathrm{C}(11)$ & $2.024(2)$ & $\mathrm{C}(25)-\mathrm{H}(25)$ & 0.93 \\
\hline $\mathrm{Fe}-\mathrm{C}(25)$ & $2.028(2)$ & $C(31)-C(36)$ & $1.380(3)$ \\
\hline $\mathrm{Fe}-\mathrm{C}(15)$ & $2.036(3)$ & $C(31)-C(32)$ & $1.396(3)$ \\
\hline $\mathrm{Fe}-\mathrm{C}(12)$ & $2.046(2)$ & $C(32)-C(33)$ & $1.383(3)$ \\
\hline $\mathrm{Fe}-\mathrm{C}(22)$ & $2.049(2)$ & $\mathrm{C}(32)-\mathrm{H}(32)$ & 0.93 \\
\hline $\mathrm{Fe}-\mathrm{C}(23)$ & $2.051(2)$ & $C(33)-C(34)$ & $1.380(4)$ \\
\hline $\mathrm{Fe}-\mathrm{C}(24)$ & $2.051(2)$ & $\mathrm{C}(33)-\mathrm{H}(33)$ & 0.93 \\
\hline $\mathrm{Fe}-\mathrm{C}(14)$ & $2.055(2)$ & $C(34)-C(35)$ & $1.374(4)$ \\
\hline \multirow[t]{2}{*}{$\mathrm{Fe}-\mathrm{C}(13)$} & $2.064(2)$ & $\mathrm{C}(34)-\mathrm{H}(34)$ & 0.93 \\
\hline & & $C(35)-C(36)$ & $1.395(3)$ \\
\hline$P(1)-C(22)$ & $1.825(2)$ & $\mathrm{C}(35)-\mathrm{H}(35)$ & 0.93 \\
\hline$P(1)-C(31)$ & $1.837(2)$ & $\mathrm{C}(36)-\mathrm{H}(36)$ & 0.93 \\
\hline$P(1)-C(41)$ & $1.843(2)$ & $C(41)-C(46)$ & $1.388(3)$ \\
\hline$P(2)-C(61)$ & $1.840(2)$ & $C(41)-C(42)$ & $1.389(3)$ \\
\hline$P(2)-C(51)$ & $1.847(2)$ & $C(42)-C(43)$ & $1.389(4)$ \\
\hline \multirow[t]{2}{*}{$P(2)-C(1)$} & $1.866(2)$ & $\mathrm{C}(42)-\mathrm{H}(42)$ & 0.93 \\
\hline & & $C(43)-C(44)$ & $1.384(5)$ \\
\hline$C(1)-C(21)$ & $1.504(3)$ & $\mathrm{C}(43)-\mathrm{H}(43)$ & 0.93 \\
\hline$C(1)-C(2)$ & $1.548(3)$ & $C(44)-C(45)$ & $1.375(5)$ \\
\hline $\mathrm{C}(1)-\mathrm{H}(1)$ & 0.98 & $\mathrm{C}(44)-\mathrm{H}(44)$ & 0.93 \\
\hline$C(2)-C(3)$ & $1.534(3)$ & $C(45)-C(46)$ & $1.393(4)$ \\
\hline $\mathrm{C}(2)-\mathrm{H}(2 \mathrm{~A})$ & 0.97 & $\mathrm{C}(45)-\mathrm{H}(45)$ & 0.93 \\
\hline $\mathrm{C}(2)-\mathrm{H}(2 \mathrm{~B})$ & 0.97 & $\mathrm{C}(46)-\mathrm{H}(46)$ & 0.93 \\
\hline$C(3)-C(11)$ & $1.501(3)$ & $C(51)-C(52)$ & $1.389(3)$ \\
\hline $\mathrm{C}(3)-\mathrm{H}(3 \mathrm{~A})$ & 0.97 & $C(51)-C(56)$ & $1.396(3)$ \\
\hline $\mathrm{C}(3)-\mathrm{H}(3 \mathrm{~B})$ & 0.97 & $C(52)-C(53)$ & $1.391(3)$ \\
\hline$C(11)-C(15)$ & $1.427(3)$ & $\mathrm{C}(52)-\mathrm{H}(52)$ & 0.93 \\
\hline$C(11)-C(12)$ & $1.432(3)$ & $C(53)-C(54)$ & $1.384(4)$ \\
\hline$C(12)-C(13)$ & $1.418(4)$ & $\mathrm{C}(53)-\mathrm{H}(53)$ & 0.93 \\
\hline $\mathrm{C}(12)-\mathrm{H}(12)$ & 0.93 & $C(54)-C(55)$ & $1.374(4)$ \\
\hline
\end{tabular}


S16

$\begin{array}{ll}\mathrm{C}(54)-\mathrm{H}(54) & 0.93 \\ \mathrm{C}(55)-\mathrm{C}(56) & 1.394(3) \\ \mathrm{C}(55)-\mathrm{H}(55) & 0.93 \\ \mathrm{C}(56)-\mathrm{H}(56 \mathrm{~A}) & 0.93 \\ \mathrm{C}(61)-\mathrm{C}(66) & 1.394(3) \\ \mathrm{C}(61)-\mathrm{C}(62) & 1.394(3) \\ \mathrm{C}(62)-\mathrm{C}(63) & 1.382(4) \\ \mathrm{C}(62)-\mathrm{H}(62 \mathrm{~A}) & 0.93 \\ \mathrm{C}(63)-\mathrm{C}(64) & 1.377(5) \\ \mathrm{C}(63)-\mathrm{H}(63 \mathrm{~A}) & 0.93 \\ \mathrm{C}(64)-\mathrm{C}(65) & 1.371(5) \\ \mathrm{C}(64)-\mathrm{H}(64) & 0.93 \\ \mathrm{C}(65)-\mathrm{C}(66) & 1.398(3) \\ \mathrm{C}(65)-\mathrm{H}(65) & 0.93 \\ \mathrm{C}(66)-\mathrm{H}(66) & 0.93 \\ \mathrm{C}(71)-\mathrm{C}(75) & 1.375(5) \\ \mathrm{C}(71)-\mathrm{C}(72) & 1.395(4) \\ \mathrm{C}(71)-\mathrm{H}(71) & 0.93 \\ \mathrm{C}(72)-\mathrm{C}(73) & 1.339(5) \\ \mathrm{C}(72)-\mathrm{H}(72) & 0.93 \\ \mathrm{C}(73)-\mathrm{C}(74) & 1.388(6) \\ \mathrm{C}(73)-\mathrm{H}(73) & 0.93 \\ \mathrm{C}(74)-\mathrm{C}(75) & 1.418(6) \\ \mathrm{C}(74)-\mathrm{H}(74) & 0.93 \\ \mathrm{C}(75)-\mathrm{H}(75) & 0.93 \\ \mathrm{O}(1)-\mathrm{C}(4) & 1.237(3) \\ \mathrm{O}(2)-\mathrm{C}(4) & 1.222(3) \\ \mathrm{C}(4)-\mathrm{C}(5) & 1.542(4) \\ \mathrm{C}(5)-\mathrm{F}(3) & 1.315(3) \\ \mathrm{C}(5)-\mathrm{F}(2) & 1.330(3) \\ \mathrm{C}(5)-\mathrm{F}(1) & 1.334(3) \\ & \end{array}$

Bond angles

$\mathrm{C}(74)-\mathrm{Ru}-\mathrm{O}(1)$

C (74) -Ru-C (73)

$O(1)-R u-C(73)$

C (74) - Ru-C (75)

$\mathrm{O}(1)-\mathrm{Ru}-\mathrm{C}(75)$

$C(73)-R u-C(75)$

$\mathrm{C}(74)-\mathrm{Ru}-\mathrm{C}(72)$

$\mathrm{O}(1)-\mathrm{Ru}-\mathrm{C}(72)$

$C(73)-R u-C(72)$

$\mathrm{C}(75)-\mathrm{Ru}-\mathrm{C}(72)$

$\mathrm{C}(74)-\mathrm{Ru}-\mathrm{C}(71)$

$\mathrm{O}(1)-\mathrm{Ru}-\mathrm{C}(71)$

C ( 73) - Ru-C (71)

C (75) - Ru-C (71)

C (72) - Ru-C (71)

C (74) - Ru-P (2)

$\mathrm{O}(1)-\mathrm{Ru}-\mathrm{P}(2)$

$C(73)-R u-P(2)$

$\mathrm{C}(75)-\mathrm{Ru}-\mathrm{P}(2)$

$\mathrm{C}(72)-\mathrm{Ru}-\mathrm{P}(2)$

$\mathrm{C}(71)-\mathrm{Ru}-\mathrm{P}$ (2)

C (74) - Ru-P (1)

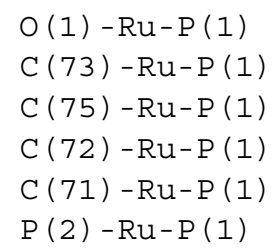

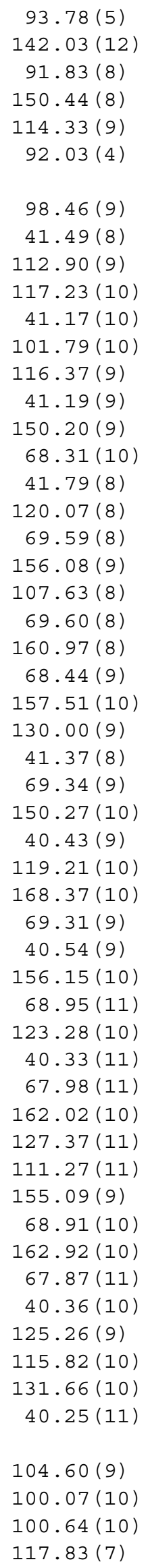




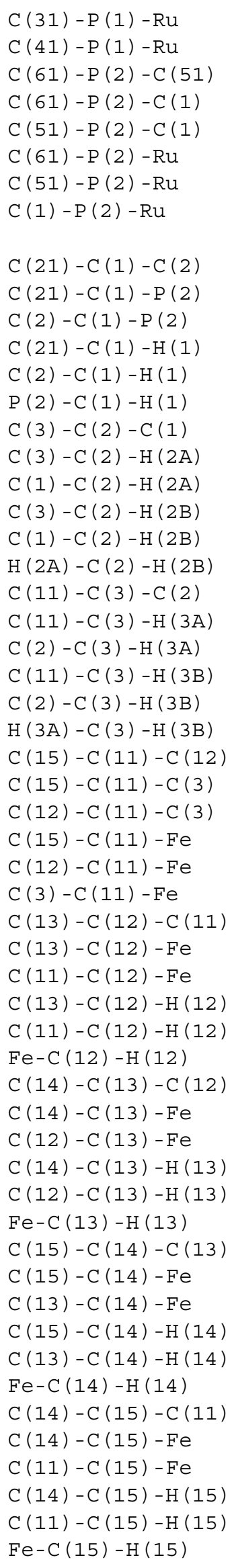

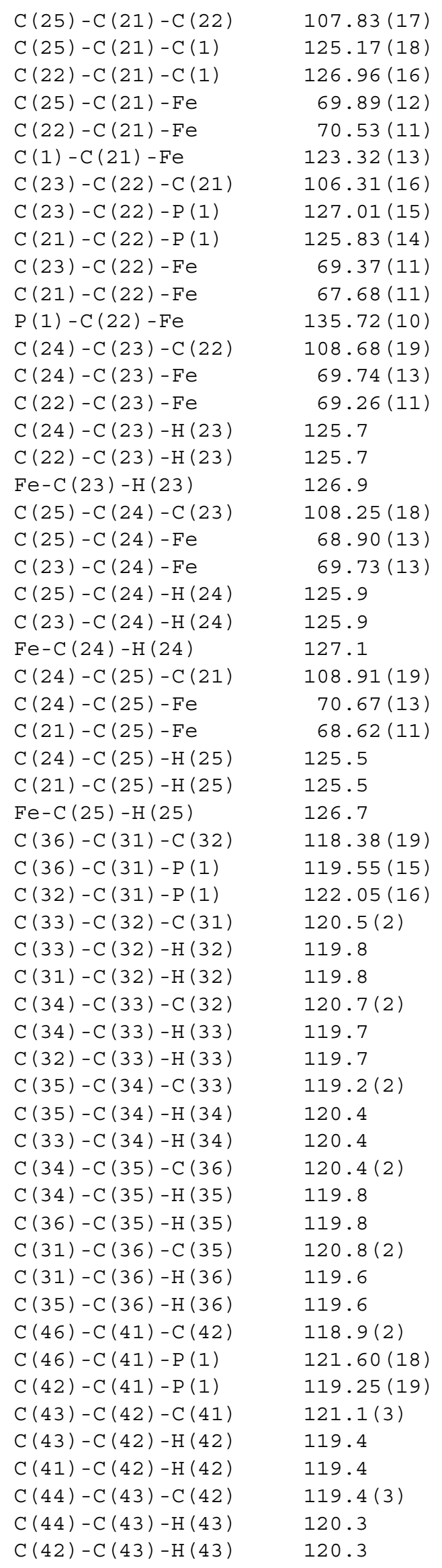




\section{S18}

$\begin{array}{ll}\mathrm{C}(45)-\mathrm{C}(44)-\mathrm{C}(43) & 120.1(3) \\ \mathrm{C}(45)-\mathrm{C}(44)-\mathrm{H}(44) & 120.0 \\ \mathrm{C}(43)-\mathrm{C}(44)-\mathrm{H}(44) & 120.0 \\ \mathrm{C}(44)-\mathrm{C}(45)-\mathrm{C}(46) & 120.6(3) \\ \mathrm{C}(44)-\mathrm{C}(45)-\mathrm{H}(45) & 119.7 \\ \mathrm{C}(46)-\mathrm{C}(45)-\mathrm{H}(45) & 119.7 \\ \mathrm{C}(41)-\mathrm{C}(46)-\mathrm{C}(45) & 119.9(3) \\ \mathrm{C}(41)-\mathrm{C}(46)-\mathrm{H}(46) & 120.1 \\ \mathrm{C}(45)-\mathrm{C}(46)-\mathrm{H}(46) & 120.1 \\ \mathrm{C}(52)-\mathrm{C}(51)-\mathrm{C}(56) & 118.1(2) \\ \mathrm{C}(52)-\mathrm{C}(51)-\mathrm{P}(2) & 122.19(17) \\ \mathrm{C}(56)-\mathrm{C}(51)-\mathrm{P}(2) & 119.23(17) \\ \mathrm{C}(51)-\mathrm{C}(52)-\mathrm{C}(53) & 120.7(2) \\ \mathrm{C}(51)-\mathrm{C}(52)-\mathrm{H}(52) & 119.6 \\ \mathrm{C}(53)-\mathrm{C}(52)-\mathrm{H}(52) & 119.6 \\ \mathrm{C}(54)-\mathrm{C}(53)-\mathrm{C}(52) & 120.4(3) \\ \mathrm{C}(54)-\mathrm{C}(53)-\mathrm{H}(53) & 119.8 \\ \mathrm{C}(52)-\mathrm{C}(53)-\mathrm{H}(53) & 119.8 \\ \mathrm{C}(55)-\mathrm{C}(54)-\mathrm{C}(53) & 119.7(2) \\ \mathrm{C}(55)-\mathrm{C}(54)-\mathrm{H}(54) & 120.1 \\ \mathrm{C}(53)-\mathrm{C}(54)-\mathrm{H}(54) & 120.1 \\ \mathrm{C}(54)-\mathrm{C}(55)-\mathrm{C}(56) & 120.0(2) \\ \mathrm{C}(54)-\mathrm{C}(55)-\mathrm{H}(55) & 120.0 \\ \mathrm{C}(56)-\mathrm{C}(55)-\mathrm{H}(55) & 120.0 \\ \mathrm{C}(55)-\mathrm{C}(56)-\mathrm{C}(51) & 121.0(2) \\ \mathrm{C}(55)-\mathrm{C}(56)-\mathrm{H}(56 \mathrm{~A}) & 119.5 \\ \mathrm{C}(51)-\mathrm{C}(56)-\mathrm{H}(56 \mathrm{~A}) & 119.5 \\ \mathrm{C}(66)-\mathrm{C}(61)-\mathrm{C}(62) & 119.0(2) \\ \mathrm{C}(66)-\mathrm{C}(61)-\mathrm{P}(2) & 120.39(17) \\ \mathrm{C}(62)-\mathrm{C}(61)-\mathrm{P}(2) & 120.57(18) \\ \mathrm{C}(63)-\mathrm{C}(62)-\mathrm{C}(61) & 120.8(3) \\ \mathrm{C}(63)-\mathrm{C}(62)-\mathrm{H}(62 \mathrm{~A}) & 119.6 \\ & \\ & \end{array}$

\begin{tabular}{|c|c|}
\hline$C(61)-C(62)-H(62 A)$ & 119.6 \\
\hline$C(64)-C(63)-C(62)$ & $119.7(3)$ \\
\hline $\mathrm{C}(64)-\mathrm{C}(63)-\mathrm{H}(63 \mathrm{~A})$ & 120.1 \\
\hline$C(62)-C(63)-H(63 A)$ & 120.1 \\
\hline$C(65)-C(64)-C(63)$ & $120.6(2)$ \\
\hline $\mathrm{C}(65)-\mathrm{C}(64)-\mathrm{H}(64)$ & 119.7 \\
\hline $\mathrm{C}(63)-\mathrm{C}(64)-\mathrm{H}(64)$ & 119.7 \\
\hline$C(64)-C(65)-C(66)$ & $120.3(3)$ \\
\hline $\mathrm{C}(64)-\mathrm{C}(65)-\mathrm{H}(65)$ & 119.8 \\
\hline $\mathrm{C}(66)-\mathrm{C}(65)-\mathrm{H}(65)$ & 119.8 \\
\hline$C(61)-C(66)-C(65)$ & $119.5(3)$ \\
\hline $\mathrm{C}(61)-\mathrm{C}(66)-\mathrm{H}(66)$ & 120.2 \\
\hline $\mathrm{C}(65)-\mathrm{C}(66)-\mathrm{H}(66)$ & 120.2 \\
\hline$C(75)-C(71)-C(72)$ & $107.8(3)$ \\
\hline$C(75)-C(71)-R u$ & $70.62(16)$ \\
\hline$C(72)-C(71)-R u$ & $71.56(14)$ \\
\hline $\mathrm{C}(75)-\mathrm{C}(71)-\mathrm{H}(71)$ & 126.1 \\
\hline $\mathrm{C}(72)-\mathrm{C}(71)-\mathrm{H}(71)$ & 126.1 \\
\hline $\mathrm{Ru}-\mathrm{C}(71)-\mathrm{H}(71)$ & 123.4 \\
\hline$C(73)-C(72)-C(71)$ & $109.0(3)$ \\
\hline$C(73)-C(72)-R u$ & $70.99(16)$ \\
\hline$C(71)-C(72)-R u$ & $71.90(14)$ \\
\hline $\mathrm{C}(73)-\mathrm{C}(72)-\mathrm{H}(72)$ & 125.5 \\
\hline $\mathrm{C}(71)-\mathrm{C}(72)-\mathrm{H}(72)$ & 125.5 \\
\hline $\mathrm{Ru}-\mathrm{C}(72)-\mathrm{H}(72)$ & 123.2 \\
\hline$C(72)-C(73)-C(74)$ & $109.2(3)$ \\
\hline$C(72)-C(73)-R u$ & $73.68(16)$ \\
\hline$C(74)-C(73)-R u$ & $69.55(17)$ \\
\hline $\mathrm{C}(72)-\mathrm{C}(73)-\mathrm{H}(73)$ & 125.4 \\
\hline $\mathrm{C}(74)-\mathrm{C}(73)-\mathrm{H}(73)$ & 125.4 \\
\hline $\mathrm{Ru}-\mathrm{C}(73)-\mathrm{H}(73)$ & 122.9 \\
\hline$C(73)-C(74)-C(75)$ & $106.7(3)$ \\
\hline
\end{tabular}


Figure. S2. Thermal ellipsoid plot (20\% ellipsoids) of $\mathrm{CpRu}\left(\mathrm{CF}_{3} \mathrm{CO}_{2}\right)\left(\mathrm{PP}^{\mathrm{Ph}} \mathrm{PF}\right),(\mathbf{1 3})$.

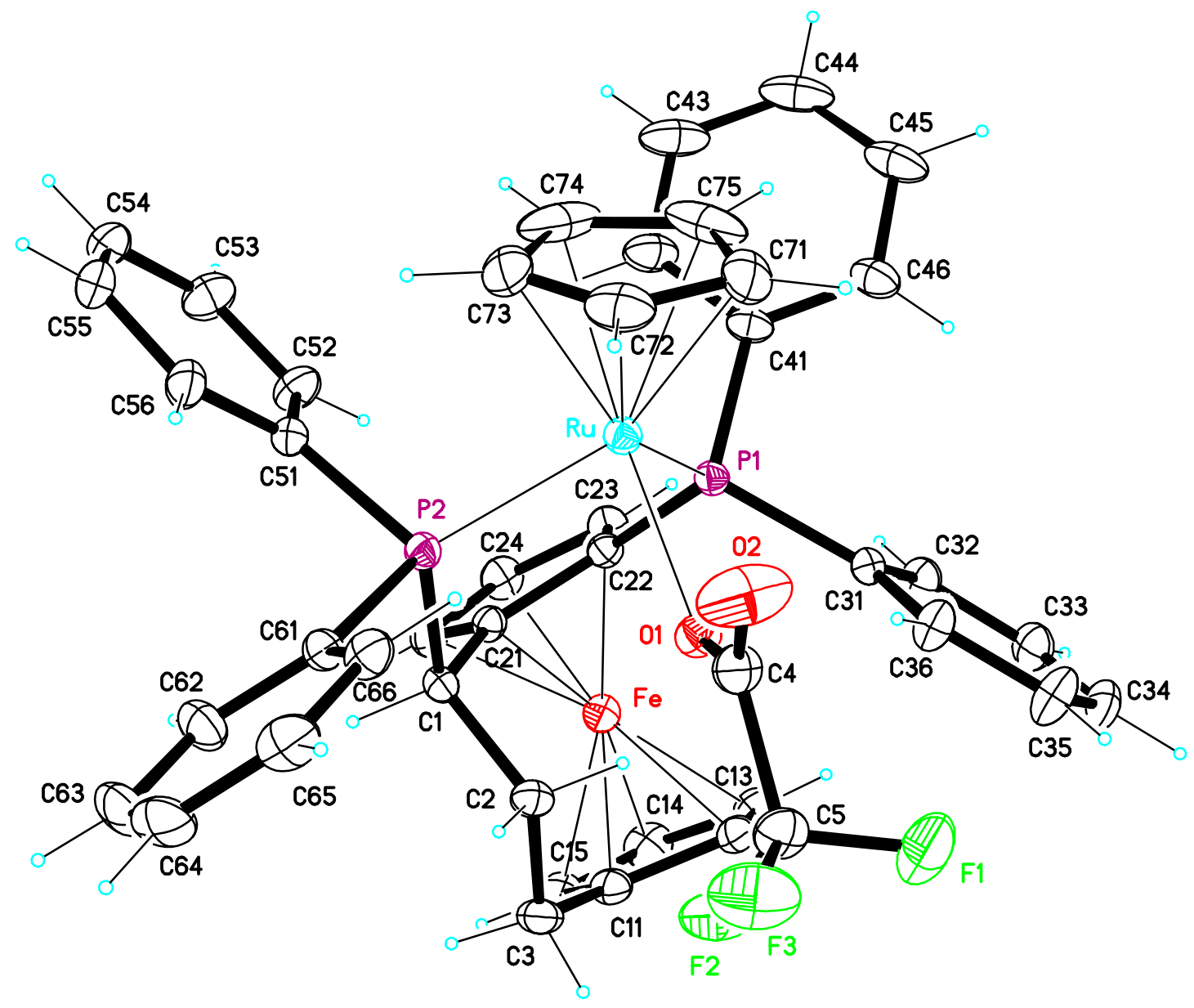




\section{${ }^{13} \mathrm{C}$ NMR information for ligands and complexes 1-6}

$\left(S_{c}, S_{p}\right)$-1-Dicyclohexylphosphino-2,1'-[1-(N,N-dimethylamino)-1,3-propanediyl]-ferrocene, $\left(\boldsymbol{S}_{c}, \boldsymbol{S}_{\boldsymbol{p}}\right)-\boldsymbol{P}^{\boldsymbol{C y}} \boldsymbol{A P F} .{ }^{13} \mathrm{C}\left\{{ }^{1} \mathrm{H}\right\}$ NMR $(75 \mathrm{MHz}$, chloroform- $d): \delta 89.45(\mathrm{Cp} C), 74.45,72.73$, 72.36, 72.28, 70.75, $69.41(\mathrm{Cp} C \mathrm{H}), 67.5\left(C^{l^{\prime \prime}}\right), 46.1\left(\mathrm{~N}\left(\mathrm{CH}_{3}\right)_{2}\right), 37.01\left(\mathrm{~d}, J_{\mathrm{CP}}=18 \mathrm{~Hz}, C^{2 "}\right)$, $39.02\left(\mathrm{~d}, J_{\mathrm{CP}}=9 \mathrm{~Hz}, \mathrm{Cy}-\mathrm{CH}\right), 36.92\left(\mathrm{~d}, J_{\mathrm{CP}}=13 \mathrm{~Hz}, \mathrm{Cy}-C \mathrm{H}\right), 28.20,28.14,27.90,27.72$, 27.62, 27.47, 27.30, 27.13, 26.97, 26.54, $\left(\mathrm{Cy}-\mathrm{CH}_{2}\right), 26.11\left(C^{3 "}\right)$.

( $\left.S_{c}, S_{p}\right)$-1-Diisopropylphosphino-2,1'-[1-(N,N-dimethylamino)-1,3-propanediyl]-ferrocene, $\left(\boldsymbol{S}_{c}, \boldsymbol{S}_{p}\right)-\boldsymbol{P}^{i p} \boldsymbol{A P} .{ }^{13} \mathrm{C}\left\{{ }^{1} \mathrm{H}\right\}$ NMR $(75 \mathrm{MHz}$, chloroform- $d): \delta 89.13,78.17,77.95(\mathrm{Cp} C), 74.16$, 72.38, 71.67, 71.29, 71.11, 69.18, $68.29(\mathrm{Cp} \mathrm{CH}), 67.07\left(C^{l "}\right), 45.54\left(\mathrm{~N}\left(\mathrm{CH}_{3}\right)_{2}\right), 37.27\left(\mathrm{~d}, J_{\mathrm{CP}}\right.$ $\left.=12 \mathrm{~Hz}, C^{2 "}\right), 26.11\left(C^{3 "}\right)$.

$\left(S_{c}, S_{p}\right)$-1-Dicyclohexylphosphino-2,1'-(1-diphenylphosphino

propanediyl)-ferrocene, $\left(\boldsymbol{S}_{c}, \boldsymbol{S}_{p}\right)-\boldsymbol{P}^{C y} \boldsymbol{P}^{\boldsymbol{P h}} \boldsymbol{P F} .{ }^{13} \mathrm{C}\left\{{ }^{1} \mathrm{H}\right\}$ NMR $(75 \mathrm{MHz}$, chloroform- $d): \delta 134.17\left(\mathrm{~d}, J_{\mathrm{CP}}=20.2 \mathrm{~Hz}\right.$, $\left.\mathrm{Ph}_{\text {ortho }}\right), 133.49\left(\mathrm{~d}, J_{\mathrm{CP}}=19.6 \mathrm{~Hz}, \mathrm{Ph}_{\text {ortho }}\right), 128.12\left(\mathrm{~d}, J_{\mathrm{CP}}=6.5 \mathrm{~Hz}, \mathrm{Ph}_{\text {meta }}\right), 127.72\left(\mathrm{~d}, J_{\mathrm{CP}}=\right.$ $\left.7.0 \mathrm{~Hz}, \mathrm{Ph}_{\text {meta }}\right), 128.85\left(\mathrm{~d}, J_{\mathrm{CP}}=25.4 \mathrm{~Hz}, \mathrm{Ph}_{\text {para }}\right), 88.66(\mathrm{Cp} \mathrm{C}), 75.60\left(\mathrm{~d}, J_{\mathrm{CP}}=14.1 \mathrm{~Hz}, \mathrm{Cp}\right.$ $C \mathrm{H}), 74.02\left(\mathrm{~d}, J_{\mathrm{CP}}=5.0 \mathrm{~Hz}, \mathrm{Cp} C \mathrm{H}\right), 74.30(\mathrm{Cp} C \mathrm{H}), 71.58\left(\mathrm{~d}, J_{\mathrm{CP}}=5.5 \mathrm{~Hz}, \mathrm{Cp} \mathrm{CH}\right), 71.42$, 70.22, $69.16(\mathrm{Cp} \mathrm{CH}), 67.58\left(C^{l^{\prime \prime}}\right), 39.22\left(\mathrm{~d}, J_{\mathrm{CP}}=16 \mathrm{~Hz}, \mathrm{Cy} C \mathrm{H}\right), 34.01\left(\mathrm{~d}, J_{\mathrm{CP}}=9 \mathrm{~Hz}, \mathrm{Cy}\right.$ $C \mathrm{H}), 32.58\left(\mathrm{~d}, J_{\mathrm{CP}}=18 \mathrm{~Hz}\right.$, Су $\left.C_{2}\right), 28.22,27.99,27.76,27.52,27.39,27.24,26.91,26.53$, 25.67, 25.52 (s, $\left.\mathrm{Cy} \mathrm{CH}_{2}\right), 25.61\left(C^{3 "}\right), 25.52\left(\mathrm{~s}, \mathrm{Cy} \mathrm{CH}_{2}\right)$.

$\left(S_{c}, S_{p}\right)$-1-Diisopropylphosphino-2,1'-(1-diphenylphosphino propanediyl)-ferrocene, $\left(S_{c}, S_{p}\right)$ $\boldsymbol{P}^{i p} \boldsymbol{P}^{\boldsymbol{P h}} \boldsymbol{P F} .{ }^{13} \mathrm{C}\left\{{ }^{1} \mathrm{H}\right\}$ NMR $(75 \mathrm{MHz}$, chloroform- $d): \delta, 133.91\left(\mathrm{~d}, J_{\mathrm{CP}}=20.9 \mathrm{~Hz}, \mathrm{Ph}_{\text {ortho }}\right)$, $133.59\left(\mathrm{~d}, J_{\mathrm{CP}}=19.1 \mathrm{~Hz}, \mathrm{Ph}_{\text {ortho }}\right), 128.32\left(\mathrm{~d}, J_{\mathrm{CP}}=6.5 \mathrm{~Hz}, \mathrm{Ph}_{\text {meta }}\right), 127.78\left(\mathrm{~d}, J_{\mathrm{CP}}=7.5 \mathrm{~Hz}\right.$, $\mathrm{Ph}_{\text {meta }}$ ), $128.74\left(\mathrm{~d}, J_{\mathrm{CP}}=25.4 \mathrm{~Hz}, \mathrm{Ph}_{\text {para }}\right), 88.35,87.64(\mathrm{Cp} \mathrm{C}), 74.26,71.43,71.12,71.03$, 69.12, 68.35, $67.46(\mathrm{Cp} C \mathrm{H}), 67.07\left(C^{l^{\prime \prime}}\right), 37.92\left(\mathrm{~d}, J_{\mathrm{CP}}=12 \mathrm{~Hz}, C^{2 "}\right), 28.01\left(\mathrm{~d}, J_{\mathrm{CP}}=9 \mathrm{~Hz}\right.$, $\left.C \mathrm{H}\left(\mathrm{CH}_{3}\right)_{2}\right), 25.82\left(\mathrm{~d}, J_{\mathrm{CP}}=13 \mathrm{~Hz}, \mathrm{CH}\left(\mathrm{CH}_{3}\right)_{2}\right), 25.71\left(C^{3 "}\right), 22.30,20.89,16.33\left(\mathrm{CH}\left(\mathrm{CH}_{3}\right)_{2}\right)$.

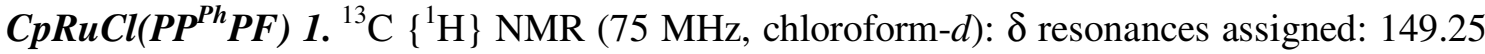
$\left(\mathrm{d}, J_{\mathrm{CP}}=28 \mathrm{~Hz}, \mathrm{Ph}_{\text {ipso }}\right), 143.51\left(\mathrm{~d}, J_{\mathrm{CP}}=24 \mathrm{~Hz}, \mathrm{Ph}_{\text {ipso }}\right), 137.22\left(\mathrm{~d}, J_{\mathrm{CP}}=14 \mathrm{~Hz}, \mathrm{Ph}_{\text {ortho }}\right)$, $136.58\left(\mathrm{~d}, J_{\mathrm{CP}}=20 \mathrm{~Hz}, \mathrm{Ph}_{\text {ortho }}\right), 134.2\left(\mathrm{~d}, J_{\mathrm{CP}}=7.5 \mathrm{~Hz}\right), 133.54,133.10,131.53,128.86$, 128.55, 127.68, $127.38\left(\mathrm{Ph}_{\text {meta }}\right.$ or $\left.\mathrm{Ph}_{\text {para }}\right), 82.12(C p \mathrm{Ru}), 88.64,82.14(C p \mathrm{Fe}-C), 79.27,73,50$, 73.42, 73.38, 72.50, 71.89, $69.26(C p \mathrm{Fe}-C \mathrm{H}), 68.86\left(\mathrm{C}^{1 "}\right), 40.87\left(\mathrm{~d}, J_{\mathrm{CP}}=13 \mathrm{~Hz}, \mathrm{C}^{2 "}\right), 25.00$ $\left(\mathrm{C}^{3}\right.$ ")

$\boldsymbol{C p R u C l}\left(\left(\boldsymbol{S}_{c}, \boldsymbol{S}_{\boldsymbol{p}}\right)-\boldsymbol{P}^{C y} \boldsymbol{P}^{\boldsymbol{P h}} \boldsymbol{P F}\right) \quad 2 .{ }^{13} \mathrm{C} \quad\left\{{ }^{1} \mathrm{H}\right\} \quad \mathrm{NMR}$ (75 MHz, chloroform- $\left.d\right)$ : $\delta$ resonances assigned: $140.50\left(\mathrm{~d}, J_{\mathrm{CP}}=42.3, \mathrm{Ph}_{\text {ipso }}\right), 135.62\left(\mathrm{~d}, J_{\mathrm{CP}}=35.2, \mathrm{Ph}_{\text {ipso }}\right), 132.22\left(\mathrm{~d}, J_{\mathrm{CP}}=11.1\right.$, $\mathrm{Ph}_{\text {orto }}$ or $\left.\mathrm{Ph}_{\text {meta }}\right), 128.42\left(\mathrm{~d}, J_{\mathrm{CP}}=8.6, \mathrm{Ph}_{\text {orto }}\right.$ or $\left.\mathrm{Ph}_{\text {meta }}\right), 124.70\left(\mathrm{~d}, J_{\mathrm{CP}}=9.6 \mathrm{Ph}_{\text {orto }}\right.$ or $\left.\mathrm{Ph}_{\text {meta }}\right)$, $124.31\left(\mathrm{~d}, J_{\mathrm{CP}}=8.6, \mathrm{Ph}_{\text {orto }}\right.$ or $\left.\mathrm{Ph}_{\text {meta }}\right), 126.46,125.92\left(\mathrm{Ph}_{\text {para }}\right), 79.30(C p \mathrm{Ru}), 89.52\left(\mathrm{dd}, J_{\mathrm{CP}}=\right.$ 16.6, 7.0, $C p \mathrm{Fe}-C), 69.94\left(\mathrm{dd}, J_{\mathrm{CP}}=27.9,9.3, C p \mathrm{Fe}-C\right), 85.05,72.90,70.58,68.52,67.80$, 67.08, 65.94, (CpFe-CH), $65.17\left(\mathrm{C}^{1 "}\right), 38.75\left(\mathrm{~d}, J_{\mathrm{CP}}=7.0 \mathrm{~Hz}, \mathrm{C}^{2}\right.$ ) $), 24.80\left(\mathrm{C}^{3}\right.$ ) $), 39.17,38.76$, $24.89,26.55,27.08,29.88,30.26,31.28(\mathrm{Cy})$.

$\boldsymbol{C p R u C l}\left(\left(\boldsymbol{S}_{\boldsymbol{c}}, \boldsymbol{S}_{\boldsymbol{p}}\right)-\boldsymbol{P}^{\text {ip }} \boldsymbol{P}^{\boldsymbol{P h}} \boldsymbol{P F}\right) 3 .{ }^{13} \mathrm{C}\left\{{ }^{1} \mathrm{H}\right\} \mathrm{MR}(75 \mathrm{MHz}$, chloroform- $d): \delta 135.73\left(\mathrm{~d}, J_{\mathrm{CP}}=11.5\right.$ $\left.\mathrm{Hz}, \mathrm{Ph}_{\text {ortho }}\right), 127.32$ (d, $J_{\mathrm{CP}}=8.5 \mathrm{~Hz}, \mathrm{Ph}_{\text {ortho }}$ or $\left.\mathrm{Ph}_{\text {meta }}\right), 131.53\left(\mathrm{~d}, J_{\mathrm{CP}}=8.2 \mathrm{~Hz}, \mathrm{Ph}_{\text {ortho }}\right.$ or $\left.\mathrm{Ph}_{\text {meta }}\right), 128.18\left(\mathrm{~d}, J_{\mathrm{CP}}=9.5 \mathrm{~Hz}, \mathrm{Ph}_{\text {ortho }}\right.$ or $\left.\mathrm{Ph}_{\text {meta }}\right), 130.00\left(\mathrm{Ph}_{\text {para }}\right), 128.42\left(\mathrm{Ph}_{\text {para }}\right), 79.52$ $(C p \mathrm{Ru}), 92.74,88.40,73.70(C p \mathrm{Fe}-C), 76.04,74.06,72.03,71.72,70.85,69.23,68.46(C p \mathrm{Fe}-$ $C \mathrm{H}), 39.60\left(\mathrm{~d}, J_{\mathrm{CP}}=9 \mathrm{~Hz}, \mathrm{C}^{2, "}\right), 38.0\left(\mathrm{~d}, J_{\mathrm{CP}}=19 \mathrm{~Hz}, \mathrm{C}^{1 "}\right), 25.05\left(\mathrm{~d}, J_{\mathrm{CP}}=12 \mathrm{~Hz}, \mathrm{C}^{3 "}\right), 32.31$ $\left(\mathrm{d}, J_{\mathrm{CP}}=27 \mathrm{~Hz}, C \mathrm{HMe}_{2}\right), 29.14\left(\mathrm{~d}, J_{\mathrm{CP}}=21.5 \mathrm{~Hz}, C \mathrm{HMe}_{2}\right), 22.04\left(\mathrm{~d}, J_{\mathrm{CP}}=7 \mathrm{~Hz}, \mathrm{CHMe} e_{2}\right.$, 
$20.79\left(\mathrm{~d}, J_{\mathrm{CP}}=5.2 \mathrm{~Hz}, \mathrm{CH} M e_{2}\right), 20.53\left(\mathrm{~d}, J_{\mathrm{CP}}=3.5 \mathrm{~Hz}, \mathrm{CH} M e_{2}\right), 17.99\left(\mathrm{~d}, J_{\mathrm{CP}}=4.3 \mathrm{~Hz}\right.$, $\left.\mathrm{CH} M e_{2}\right)$.

CpRuH(PP $\left.{ }^{P h} \boldsymbol{P F}\right)$ 4. ${ }^{13} \mathrm{C}\left\{{ }^{1} \mathrm{H}\right\}$ NMR $\left(75 \mathrm{MHz}\right.$, acetone- $\left.d_{6}\right): \delta$ resonances assigned 142.40, 143.3, $150.9\left(\mathrm{Ph}_{\text {ipso }}\right), 126.44\left(\mathrm{~d}, J_{\mathrm{CP}}=9 \mathrm{~Hz}, \mathrm{Ph}_{\text {meta }}\right), 127.42\left(\mathrm{~d}, J_{\mathrm{CP}}=6.5, \mathrm{Ph}_{\text {meta }}\right), 127.83(\mathrm{~d}$, $\left.J_{\mathrm{CP}}=7 \mathrm{~Hz}, \mathrm{Ph}_{\text {meta }}\right), 128.74\left(\mathrm{~d}, J_{\mathrm{CP}}=25 \mathrm{~Hz}, \mathrm{Ph}_{\text {ortho }}\right), 129.28,130.51,131.42\left(\mathrm{Ph}_{\text {para }}\right), 82.19$ $(C p \mathrm{Ru}), 93.50,72.80(C p \mathrm{Fe}-C), 87.99,76.12,73.11,73.01,72.12,71.86,69.18(C p \mathrm{Fe}-C \mathrm{H})$, $68.44\left(\mathrm{C}^{1 "}\right), 35.67\left(\mathrm{~d}, J_{\mathrm{CP}}=12 \mathrm{~Hz}, \mathrm{C}^{2}\right), 24.79\left(\mathrm{C}^{3,}\right)$.

CpRuH $\left(\left(\boldsymbol{S}_{c}, S_{l}\right) \boldsymbol{P}^{C y} \boldsymbol{P}^{\boldsymbol{P h}} \boldsymbol{P F}\right)$ 5. ${ }^{13} \mathrm{C}\left\{{ }^{1} \mathrm{H}\right\}$ NMR (75 MHz, benzene- $\left.d_{6}\right)$ : $\delta$ resonances assigned: $134.63\left(\mathrm{~d}, J_{\mathrm{CP}}=20 \mathrm{~Hz}, \mathrm{Ph}_{\text {ortho }}\right), 134.15\left(\mathrm{~d}, J_{\mathrm{CP}}=19 \mathrm{~Hz}, \mathrm{Ph}_{\text {ortho }}\right), 127.45\left(\mathrm{~d}, J_{\mathrm{CP}}=7.5 \mathrm{~Hz}\right.$, $\left.\mathrm{Ph}_{\text {para }}\right), 127.65\left(\mathrm{~d}, J_{\mathrm{CP}}=8.0 \mathrm{~Hz}, \mathrm{Ph}_{\text {para }}\right), 128.58\left(\mathrm{~d}, J_{\mathrm{CP}}=16 \mathrm{~Hz}, \mathrm{Ph}_{\text {meta }}\right), 129.72\left(\mathrm{~d}, J_{\mathrm{CP}}=18\right.$ $\left.\mathrm{Hz}, \mathrm{Ph}_{\text {meta }}\right), 80.15(C p \mathrm{Ru}), 87.78(C p \mathrm{Fe}-C), 80.25,75.54,73.70,71.03,69.12,68.35,67.46$ $(C p \mathrm{Fe}-\mathrm{CH}), 67.95\left(\mathrm{C}^{1 "}\right), 41.78\left(\mathrm{~d}, J_{\mathrm{CP}}=8.0 \mathrm{~Hz}, \mathrm{C}^{2}\right.$ '), $26.40\left(\mathrm{C}^{3,}\right), 24.64-35.45(\mathrm{Cy})$.

$\boldsymbol{C p R u H}\left(\left(\boldsymbol{S}_{c}, \boldsymbol{S}_{\boldsymbol{p}}\right) \boldsymbol{P}^{\text {ip }} \boldsymbol{P}^{\boldsymbol{P h}} \boldsymbol{P F}\right) \boldsymbol{6} .{ }^{13} \mathrm{C}\left\{{ }^{1} \mathrm{H}\right\}$ NMR $\left(75 \mathrm{MHz}\right.$, acetone- $\left.d_{6}\right)$ : $\boldsymbol{\delta}$ resonances assigned: 6M: $136.40\left(\mathrm{~d}, J_{\mathrm{CP}}=11 \mathrm{~Hz}, \mathrm{Ph}_{\text {ortho }}\right), 132.27\left(\mathrm{~d}, J_{\mathrm{CP}}=9 \mathrm{~Hz}, \mathrm{Ph}_{\text {ortho }}\right), 130.40\left(\mathrm{~d}, J_{\mathrm{CP}}=2.1\right.$, $\mathrm{Ph}_{\text {meta }}$ or $\left.\mathrm{Ph}_{\text {para }}\right), 128.32\left(\mathrm{~d}, J_{\mathrm{CP}}=10.2 \mathrm{~Hz}, \mathrm{Ph}_{\text {meta }}\right.$ or $\left.\mathrm{Ph}_{\text {para }}\right), 127.85\left(\mathrm{~d}, J_{\mathrm{CP}}=2.5 \mathrm{~Hz}, \mathrm{Ph}_{\text {meta }}\right.$ or $\left.\mathrm{Ph}_{\text {para }}\right), 124.03\left(\mathrm{~d}, J_{\mathrm{CP}}=2.0, \mathrm{Ph}_{\text {meta }}\right.$ or $\left.\mathrm{Ph}_{\text {para }}\right), 80.06(C p \mathrm{Ru}), 94.21,84.92,76.50,74.43$, 72.20, 72.10, 71.01, $69.77(C p F e), 69.13\left(\mathrm{~d}, J_{\mathrm{CP}}=4.1 \mathrm{~Hz}, \mathrm{C}^{1 "}\right), 39.53\left(\mathrm{~d}, J_{\mathrm{CP}}=6.8 \mathrm{~Hz}, \mathrm{C}^{2}\right.$ "), $37.90\left(\mathrm{~d}, J_{\mathrm{CP}}=19.0 \mathrm{~Hz}, C \mathrm{HMe}_{2}\right), 29.75\left(\mathrm{~d}, J_{\mathrm{CP}}=22.0 \mathrm{~Hz}, C \mathrm{HMe}_{2}\right), 25.08\left(\mathrm{~d}, J_{\mathrm{CP}}=11 \mathrm{~Hz}\right.$, $\mathrm{C}^{3 "}$ ), $22.41\left(\mathrm{~d}, J_{\mathrm{CP}}=7.6, \mathrm{CHMe} e_{2}\right), 20.82\left(\mathrm{~d}, J_{\mathrm{CP}}=5.0 \mathrm{~Hz}, \mathrm{CH} M e_{2}\right), 20.58\left(\mathrm{~d}, J_{\mathrm{CP}}=3.5 \mathrm{~Hz}\right.$, $\mathrm{CHMe} 2), 18.25$ (d, $\left.J_{\mathrm{CP}}=5.0 \mathrm{~Hz}, \mathrm{CHMe} e_{2}\right) .6 \mathrm{~m}: 134.32$ (d, $\left.J_{\mathrm{CP}}=11 \mathrm{~Hz}, \mathrm{Ph}_{\text {ortho }}\right), 134.07$ (d, $J_{\mathrm{CP}}$ $\left.=11 \mathrm{~Hz}, \mathrm{Ph}_{\text {ortho }}\right), 129.35\left(\mathrm{~d}, J_{\mathrm{CP}}=2.0, \mathrm{Ph}_{\text {meta }}\right.$ or $\left.\mathrm{Ph}_{\text {para }}\right), 128.87\left(\mathrm{~d}, J_{\mathrm{CP}}=2.0, \mathrm{Ph}_{\text {meta }}\right.$ or $\left.\mathrm{Ph}_{\text {para }}\right), 127.68\left(\mathrm{~d}, J_{\mathrm{CP}}=9.0 \mathrm{~Hz}, \mathrm{Ph}_{\text {meta }}\right.$ or $\left.\mathrm{Ph}_{\text {para }}\right), 127.63\left(\mathrm{~d}, J_{\mathrm{CP}}=9.0 \mathrm{~Hz}, \mathrm{Ph}_{\text {meta }}\right.$ or $\left.\mathrm{Ph}_{\text {para }}\right)$, $79.52(C p \mathrm{Ru}), 74.13,71.59,71.09,68.70(C p \mathrm{Fe}), 67.85\left(\mathrm{~d}, J_{\mathrm{CP}}=4.5 \mathrm{~Hz}, \mathrm{C}^{1}{ }^{\prime \prime}\right), 41.72\left(\mathrm{~d}, J_{\mathrm{CP}}=\right.$ $\left.8.5 \mathrm{~Hz}, \mathrm{C}^{2 \prime}\right), 24.50\left(\mathrm{~d}, J_{\mathrm{CP}}=12 \mathrm{~Hz}, \mathrm{C}^{3 "}\right), 20.51\left(\mathrm{~d}, J_{\mathrm{CP}}=5.5, \mathrm{CH} \mathrm{Me}_{2}\right), 20.33\left(\mathrm{~d}, J_{\mathrm{CP}}=5.6 \mathrm{~Hz}\right.$, $\mathrm{CHMe} 2$ ), $20.07\left(\mathrm{~d}, J_{\mathrm{CP}}=5.2 \mathrm{~Hz}, \mathrm{CHMe} 2\right), 19.08\left(\mathrm{~d}, J_{\mathrm{CP}}=4.9 \mathrm{~Hz}, \mathrm{CH} M e_{2}\right)$. 